\title{
Electron-deuteron scattering in the equal-time formalism: beyond the impulse approximation
}

\author{
D. R. Phillips ${ }^{a *, b}$, S. J. Wallace ${ }^{c *, d}$, N. K. Devine ${ }^{e}$ \\ ${ }^{a}$ Department of Physics and Astronomy, Ohio University, Athens, OH 45701 \\ ${ }^{b}$ Department of Physics, University of Washington, Box 351560, Seattle, WA 98190 \\ ${ }^{c}$ Department of Physics, University of Maryland, College Park, Maryland 20742-4111 \\ ${ }^{d}$ Theory Division, Jefferson Laboratory, 12000 Jefferson Avenue, Newport News, VA 23606 \\ ${ }^{e}$ General Sciences Corporation, 4600 Powder Mill Rd., Suite 400, Beltsville, Maryland 20705-1675
}

\begin{abstract}
Using a three-dimensional formalism that includes relativistic kinematics, the effects of negativeenergy states, approximate boosts of the two-body system, and current conservation, we calculate the electromagnetic form factors of the deuteron up to $Q^{2}$ of $4 \mathrm{GeV}^{2}$. This is done using a dynamical boost for two-body systems with spin. We first compute form factors in impulse approximation, but then also add an isoscalar meson-exchange current of pion range that involves the $\gamma \pi$ contact operator associated with pseudovector $\pi N$ coupling. We also consider effects of the $\rho \pi \gamma$ mesonexchange current. The experimentally measured quantities $A, B$, and $t_{20}$ are calculated over the kinematic range probed in recent Jefferson Laboratory experiments. The $\rho \pi \gamma$ meson-exchange current provides significant strength in $A$ at large $Q^{2}$ and the $\gamma \pi$ contact-term exchange current shifts $t_{20}$, providing good agreement with the JLab data. Relativistic effects and the $\gamma \pi$ meson exchange current do not provide an explanation of the $B$ observable, but the $\rho \pi \gamma$ current could help to provide agreement if a nonstandard value is used for the tensor $\rho N$ coupling that enters this contribution.
\end{abstract}

\section{INTRODUCTION}

The deuteron is a bound state of two spin- $1 / 2$ particles. Consequently, the combined dictates of Poincaré invariance, current conservation, and parity tell us that it has three independent form factors. Conventionally these are taken to be the deuteron charge, magnetic, and quadrupole form factors, $G_{C}, G_{M}$, and $G_{Q}$, which are related to the Breit-frame matrix elements of the deuteron electromagnetic current operator $\mathcal{A}_{\mu}$ in the three deuteron magnetic sub-states $|+1\rangle,|0\rangle$, and $|-1\rangle$ via the formulae:

$$
\begin{aligned}
G_{C} & =\frac{1}{3 \sqrt{1+\eta} e}\left(\left\langle 0\left|\mathcal{A}_{0}\right| 0\right\rangle+2\left\langle+1\left|\mathcal{A}_{0}\right|+1\right\rangle\right), \\
G_{Q} & =\frac{1}{2 \eta \sqrt{1+\eta} e}\left(\left\langle 0\left|\mathcal{A}_{0}\right| 0\right\rangle-\left\langle+1\left|\mathcal{A}_{0}\right|+1\right\rangle\right), \\
G_{M} & =\frac{-1}{\sqrt{2 \eta(1+\eta)} e}\left\langle+1\left|\mathcal{A}_{+}\right| 0\right\rangle .
\end{aligned}
$$

(Here $\eta=Q^{2} /\left(4 M_{d}^{2}\right)$, and $Q^{2}=-q^{2}$ is the absolute value of the square of the four-momentum transfer to the deuteron.) Three experimental quantities are therefore required to disentangle the electromagnetic current of this nucleus. Two of these - the structure functions $A$ and $B$ - can be obtained from the electron-deuteron differential cross-section using the usual Rosenbluth separation:

$$
\frac{d \sigma}{d \Omega}=\frac{d \sigma}{d \Omega} \text { Mott }\left[A\left(Q^{2}\right)+B\left(Q^{2}\right) \tan ^{2}\left(\frac{\theta_{e}}{2}\right)\right] .
$$

The structure functions $A$ and $B$ are related to $G_{C}, G_{Q}$, and $G_{M}$, as follows:

$$
A=G_{C}^{2}+\frac{8}{9} \eta^{2} G_{Q}^{2}+\frac{2}{3} \eta G_{M}^{2}
$$

$$
B=\frac{4}{3} \eta(1+\eta) G_{M}^{2} .
$$

The third observable of choice is the dependence of the scattering on the (tensor) polarization of deuterium. This can be measured either using a polarized deuteron target (and unpolarized beam), or with an unpolarized target by measuring the polarization of the recoil deuterons. Both types of experiment result in the same tensor-polarization observable, and in particular both can measure:

$$
t_{20} \equiv-\sqrt{2} \frac{x(x+2)+y / 2}{1+2\left(x^{2}+y\right)} ;
$$

where:

$$
x=\frac{2 \eta G_{Q}}{3 G_{C}} ; y=\frac{2 \eta}{3}\left[\frac{1}{2}+(1+\eta) \tan ^{2}\left(\frac{\theta_{e}}{2}\right)\right] \frac{G_{M}^{2}}{G_{C}^{2}} .
$$

Recent experiments at the Thomas Jefferson National Accelerator Facility (JLab) have probed the electromagnetic form factors of the deuteron at large space-like momentum transfers. $t_{20}$ has been measured at $Q^{2}$ up to almost $2 \mathrm{GeV}^{2}$ [1, 2], $B$ out to about $1.3 \mathrm{GeV}^{2}$, and $A$ to $Q^{2}=6 \mathrm{GeV}^{2}$ [3, 4]. Two recent reviews provide up-to-date information on these experiments and their theoretical interpretation [5, []] .

The kinematic range of these data pushes the limit of theoretical descriptions of this simplest of nuclei. At small values of $Q^{2}$ the appropriate degrees of freedom for this description are nonrelativistic nucleons, interacting via static nucleon-nucleon interactions, with small corrections due to meson-exchange currents and relativistic effects [7, 8, 9, 10, 11]. Provided $Q^{2}$ is below the scale of chiral symmetry breaking, contributions to electrondeuteron scattering can be organized using the nuclear 
effective theory first proposed by Weinberg [12, 13, 14], and the results are in good agreement with the extant data 15 below $Q^{2} \sim 0.5 \mathrm{GeV}^{2}$. As $Q^{2}$ increases to $\sim 1 \mathrm{GeV}^{2}$, nucleon and meson degrees of freedom may still be appropriate but a relativistic formalism is needed to account for relativistic kinematics and dynamics. At very high values of $Q^{2}$ one expects a transition to a regime where quark and gluon degrees of freedom provide the most natural description of data. To date, the experimental and theoretical situation for elastic electrondeuteron scattering can be summarized by saying that there is no significant evidence of having reached the quark regime [5]. The most important aspects of the internal quark structure of a nucleon appear to be taken into account via nucleon form factors and relativistic $N N$ dynamics.

Considerable effort has been invested in the construction of these relativistic formalisms for the $N N$ bound state and associated meson-exchange currents. The goal is to address elastic electron-deuteron scattering at $Q^{2}$ of a few $\mathrm{GeV}^{2}$, see e.g. Refs. 16, 17, 18, 19, 20, 21, 22, 23]. Such an approach is a logical extension of the standard nonrelativistic treatment of the $N N$ system (see, e.g. Ref. 24]) which has had a significant amount of success in describing $A, B$, and $t_{20}$ using a non-relativistic $N N$ interaction that is fit to the $N N$ scattering data (see, e.g. Refs. 7, 8, 9]). Like the non-relativistic approach, relativistic approaches to the $N N$ problem are grounded in a phenomenological description of the $N N$ scattering data. However, they seek to implement dynamics that obeys the Poincaré algebra - even if only approximately - and in so doing they go beyond the nonrelativistic treatment of the $N N$ system. If electromagnetic interactions with the deuteron are also to be considered then-regardless of the momentum transfer involved-it is crucial that the consequences of electromagnetic gauge invariance be incorporated in the calculation. Minimally this means that the electromagnetic current of the deuteron must be conserved. Indeed, the derivation of Eqs. (11)-(3) assumed that the deuteronic current $\mathcal{A}_{\mu}$ was conserved. This motivates the use of meson-exchange interactions for which methods of quantum field theory may be used to construct the conserved current.

The three-dimensional "equal-time" (ET) formalism which was developed and applied in Refs. 16, 17, 18] is one such method. This approach starts from the fourdimensional Bethe-Salpeter formalism and the Mandelstam construction of the electromagnetic current. It includes relativistic kinematics, negative-energy states and relativistic pieces of the electromagnetic current explicitly at all stages of the calculation. In Ref. [18] we reported on impulse-approximation calculations of the form factors (11)-(3). The Bonn-B interaction 25], that was fit to $N N$ scattering data using a relativistic equation with only positive-energy states, was used. In contrast to Ref. 25] we employed pseudovector $\pi N$ coupling, since it is then easier to implement the constraints of chi- ral symmetry. In Ref. [18] we focused particularly on the role of negative-energy components of the deuteron wave function in the ET formalism. The $N N$ interaction with negative-energy components was obtained by adjusting the $\sigma N N$ coupling so that the deuteron binding energy was the same as in Ref. 25]. Once this was done the inclusion of negative-energy components of spinors produced only modest effects: there were noticeable changes to some deuteron observables at larger momentum transfers but not much improvement in the description of the experimental data. This is in agreement with earlier results of Hummel and Tjon [19], and is not surprising since pseudovector coupling suppresses the coupling to the negative-energy states.

The results of Ref. 18 for observable $t_{20}$ were in good agreement with experiments but the description of the deuteron magnetic form factor was poor. Similar conclusions held regardless of whether or not the negative-energy components of the deuteron wave function were included. Some other relativistic approaches, e.g. Ref. 20], have found larger effects for negative-energy components and this point is discussed further in Sec. IX]

However, a number of important effects were not included in the impulse-approximation calculation of Ref. [18] (hereafter "ETIA"). These included the dynamical boost of bound-state vertex functions, an isoscalar pionic meson-exchange contribution to $\mathcal{A}_{\mu}$ that arises from use of pseudovector $\pi N$ coupling, and the $\rho \pi \gamma$ meson-exchange current. In the present work we use the positive-energy-state ETIA calculation as a baseline. We then include the various effects listed above that were omitted in Ref. 18], and also display the contribution of Z-graphs computed to first order in perturbation theory.

These different contributions to $\mathcal{A}_{\mu}$ can be organized according to the power counting developed by Weinberg [12, 13, 14] that has already been successfully applied to a number of electromagnetic reactions involving deuterium. Since the power counting is based on an expansion in powers of

$$
\mathcal{P} \equiv \frac{|\vec{p}|, m_{\pi},|\vec{q}|}{\Lambda},
$$

with $\Lambda$ the scale of chiral symmetry breaking, it breaks down at $Q^{2} \sim 0.5 \mathrm{GeV}^{2}$, but examining the different contributions to $\mathcal{A}_{\mu}$ using the nuclear effective theory provides a way to anchor their low-momentum behavior in a systematic way.

In this counting the calculation of Ref. [18] included all mechanisms of $O(e)$ and $O(e \mathcal{P})$. Formally the most important neglected mechanism occurs at $O\left(e \mathcal{P}^{2}\right)$, and arises because electromagnetic currents are evaluated in the Breit frame, where the deuteron has momentum $-\frac{1}{2} \mathbf{q}$ in the initial state and momentum $+\frac{1}{2} \mathbf{q}$ in the final state. In this case the deuteron wave functions must be boosted from the deuteron rest frame to obtain the initial and final-state wave functions in the Breit frame. The ET formalism developed in Ref. 16, 17, 18] was not boost invariant, and this failure to respect Poincaré invariance re- 
sulted in an error at $O\left(e \mathcal{P}^{2}\right)$ in the calculation of Ref. [18]. In order to remedy this, a boost rule for scalar particles was developed in Ref. 26] where it was shown to provide an exact result for the boost of the two-body energy. In this work, the boost rule is extended approximately to the case of two interacting spin- $1 / 2$ particles as discussed in Sec. D We have verified numerically that the ET equation together with the boost rule for the $N N$ interaction provides eigenstates of the deuteron with energy $E_{\mathbf{P}}=\sqrt{M^{2}+\mathbf{P}^{2}}$ when the total momentum is $\mathbf{P}$. The deuteron mass $M$ is therefore invariant for the total momentum values of interest in this work.

An exact solution of the boost problem has been given in the instant form of dynamics by Coester and Polyzou [27], following the methods suggested by Bakamjian and Thomas 28]. In that solution, interactions are introduced solely into the rest-frame mass operator. In contrast, here we use the ET reduction of field-theoretic expressions to find an effective hamiltonian as well as currents that are consistent with that hamiltonian. (In particular, we ensure that the currents satisfy a WardTakahashi identity.) In the two-body rest-frame system, the hamiltonian found via our ET reduction could be used to define a suitable mass operator, which could then be used to implement an exact boost to other frames following the methods of Ref. [27]. We do not employ this "exact boost" construction because the electromagnetic currents that are obtained from the ET reduction are not consistent with it. Instead, in our work we develop an approximate boost rule which maintains a clear correspondence to field theory, and so facilitates a more consistent treatment of strong and electromagnetic interactions.

Another important contribution not included in Ref. 18 enters at $O\left(e \mathcal{P}^{3}\right)$. When pseudovector (PV) $\pi N$ coupling is employed an additional contribution to the two-body isoscalar charge operator - first identified by Riska 29] - must be included. Without it the unitary equivalence between PV and pseudoscalar $\pi N$ coupling will not be respected [30. In this work we include this $\mathrm{PV}$-coupling current and find that it is significant, albeit not as important as when it is added in some nonrelativistic approaches [7]. The decreased importance of this effect in our approach is traceable to a factor of half in the PV-coupling current in the ET formalism relative to the current used in Ref. 7]. This factor must be taken into account if the unitary equivalence between different techniques for obtaining relativistic corrections to the $N N$ interaction and currents is to be maintained [31].

The $\rho \pi \gamma$ meson-exchange current (MEC) has often been invoked in calculations of electron-deuteron scattering. In this MEC the photon interacts with the $\rho$ meson cloud of one nucleon, producing a pion which is absorbed by the other nucleon. Thus the $\gamma N$ interaction is of short range but the MEC has long range because a pion exchange is involved. This MEC contributes to $G_{M}$ at $O\left(e \mathcal{P}^{4}\right)$ in the nuclear effective theory, but as we show it can produce substantial effects in the magnetic form factor at large $Q$. We include it in our relativis- tic calculation and consider the extent to which it can help to explain the magnetic form factor in the ET formalism. Although there is not much improvement in the description of data if the standard tensor $\rho N$ coupling of one-boson-exchange $N N$ models is adopted, other values of $f_{\rho} / g_{\rho}$ may help to explain $B\left(Q^{2}\right)$. Note that we do not include the $\omega \sigma \gamma$ current that has been considered by Hummel and Tjon [19] because it is of shorter range.

Our paper is structured as follows. First we give a brief review of the ET formalism in which we display expressions for the bound-state equation, the $N N$ interaction, and the current matrix element-all for the particular case of an instantaneous two-body interaction. Second, we discuss the boost of the bound state wave function that is needed to evaluate matrix elements in the Breit frame. Third, we discuss inclusion of meson-exchangecurrent contributions, especially the PV-coupling current and the $\rho \pi \gamma$ MEC, each of which has been found to give significant contributions to electron-deuteron scattering. We then also describe the inclusion of Z-graph effects (coupling to the negative-energy states) in perturbation theory. Lastly, we present our results for $A, B$, and $t_{20}$ when each of these effects is added to the baseline ETIA calculation.

\section{THE EQUAL-TIME APPROACH}

A number of alternative 3D relativistic treatments of deuteron dynamics exist (see, for instance, Refs. 19, 20, $21,[22,23])$. Of these, the formalism that is closest to this work is that of Hummel and Tjon [19], although here we eliminate some approximations made in Ref. [19]. In this section we summarize the development of the ET formalism with respect to obtaining the one-body limit and a systematic reduction from $4 \mathrm{D}$ to $3 \mathrm{D}$.

Consider the 4D Bethe-Salpeter equation (BSE) for a bound-state vertex function, $\Gamma$ :

$$
\Gamma=K G_{0} \Gamma
$$

Here $K$ is, in principle, the sum of all two-particleirreducible $N N \rightarrow N N$ graphs. The $N N$ propagator $G_{0}$ is the product of spin-half Feynman propagators for each nucleon: $G_{0}=i d_{1} d_{2}$. In studies of this equation for the deuteron bound state 32 the kernel $K$ included a set of single-boson exchanges - in analogy to many nonrelativistic potential models - yielding the "ladder" approximation. However, it is well known that in such an approximation the Bethe-Salpeter equation does not give the correct one-body limit [33]. In other words, if we consider unequal-mass particles, and take one of them to be very heavy, Eq. (10) does not become the Dirac equation for the light particle moving in the static field of the heavy one. This limit is only properly treated in Eq. (10) if the full set of ladder and crossed-ladder graphs is taken for $K$ 33]. In Ref. 16] we provided a remedy to this flaw, and showed that the pieces of the graphs that appear in 
$K$ and are responsible for the one-body limit can be resummed so that Eq. (10) becomes:

$$
\Gamma=U\left(G_{0}+G_{C}\right) \Gamma
$$

where the precise form of $G_{C}$ was derived in 16, 18]. For exact correspondence between (10) and (11) we should have:

$$
K=U+U G_{C} K
$$

At the level of the one-boson-exchange interaction, where $K$ and $U$ have only their lowest-order pieces, we see that Eq. (11) defines an improved "ladder" Bethe-Salpeter equation, which does have the correct one-body limit:

$$
\Gamma=K^{(2)} G \Gamma
$$

where $G=G_{0}+G_{C}$. Note that if the field theory to be solved involves nucleons and mesons then the approximation $K \rightarrow K^{(2)}$ is equivalent to restricting ourselves to a one-boson-exchange kernel.

This equation is still four-dimensional. The next step is to perform a systematic reduction to three dimensions. We can motivate the reduction scheme by following Salpeter [34] and assuming that the dominant interaction is the instantaneous part, i.e., the part obtained by the replacement:

$$
K^{(2)}(q)=\frac{\Gamma_{1} \Gamma_{2}}{q^{2}-\mu^{2}} \quad \longrightarrow \quad K_{\text {inst }}^{(2)}(\mathbf{q})=-\frac{\Gamma_{1} \Gamma_{2}}{\mathbf{q}^{2}+\mu^{2}}
$$

where $\Gamma_{1}$ and $\Gamma_{2}$ are Dirac-matrix structures of the meson-nucleon vertices and $q=\left(q_{0}, \mathbf{q}\right)$ is the fourmomentum of the meson. Since $K_{\text {inst }}^{(2)}$ depends only on the three-vector $\mathbf{q}$ this replacement reduces Eq. (13) to a three-dimensional equation. If we were to stop at this point, the reduction would not be systematic nor would it include retardation effects. A central result of Ref. 16 was that the three-dimensional reduction could be implemented in a systematically improvable way. Instead of simply neglecting the retardation effects, they can be reorganized into a $3 \mathrm{D}$ interaction kernel $K_{\mathrm{ET}}$. This interaction shares the property of $K_{\text {inst }}^{(2)}$ that it does not depend upon the time-component of momentum transfer. If the reduction includes only positive-energy matrix elements, then $K_{\mathrm{ET}}$ is the 3D interaction obtained from time-ordered perturbation theory.

Thus the reduction to three dimensions produces the following two-body equation:

$$
\Gamma_{\mathrm{ET}}=K_{\mathrm{ET}}\langle G\rangle \Gamma_{\mathrm{ET}}
$$

Here the three-dimensional propagator $\langle G\rangle$ is obtained by integrating over the time-component of relative fourmomentum:

$$
\langle G\rangle \equiv \int \frac{d p_{0}}{2 \pi}\left[G_{0}(p ; P)+G_{C}(p ; P)\right]
$$

and the ET interaction is defined in lowest order by

$$
\langle G\rangle K_{\mathrm{ET}}\langle G\rangle \equiv\langle G K G\rangle
$$

(Hereafter we always denote integration over zeroth components of relative four-momenta by angled brackets.)

In order to explain the significance of the $G_{C}$ term in $\langle G\rangle$ and its connection to $\mathrm{Z}$ graphs and the one-body limit, consider the more standard equal-time Green's function [35, 36] that omits $G_{C}$. It is sufficient to work in the c.m. frame of two particles of equal masses because the boost discussed in Sec. $\nabla$ will provide the results needed in other frames. Then one finds

$$
\left\langle G_{0}\right\rangle=\frac{\Lambda_{1}^{+} \Lambda_{2}^{+}}{E-2 \epsilon}-\frac{\Lambda_{1}^{-} \Lambda_{2}^{-}}{E+2 \epsilon},
$$

where $\Lambda^{ \pm}$are related to projection operators onto positive and negative-energy states of the Dirac equation, $E$ is the total energy, and $\epsilon=\left(\mathbf{p}^{2}+m^{2}\right)^{1 / 2}$. The propagator $\left\langle G_{0}\right\rangle$ is not invertible [37], since it has no components in the +- and -+ sectors. This is related to the lack of a correct one-body limit in the ladder BSE. If we had applied the 3D reduction (14) to Eq. (10) we would have obtained the Salpeter equation:

$$
\Gamma_{\mathrm{S}}=K_{\mathrm{inst}}\left\langle G_{0}\right\rangle \Gamma_{\mathrm{S}}
$$

which has the non-invertible $\left\langle G_{0}\right\rangle$ in the intermediate state. However, adding $G_{C}$ - which comes from resumming pieces of the crossed-ladder graphs - before reducing to three dimensions gives a $3 \mathrm{D} N N$ propagator:

$$
\langle G\rangle=\frac{\Lambda_{1}^{+} \Lambda_{2}^{+}}{E-2 \epsilon}-\frac{\Lambda_{1}^{+} \Lambda_{2}^{-}}{2 \epsilon}-\frac{\Lambda_{1}^{-} \Lambda_{2}^{+}}{2 \epsilon}-\frac{\Lambda_{1}^{-} \Lambda_{2}^{-}}{E+2 \epsilon} .
$$

This is the three-dimensional propagator that was derived by Mandelzweig and Wallace [38], here specialized to the c.m. frame for two equal-mass particles. In its more general form for unequal masses, the contribution $\left\langle G_{C}\right\rangle$ to $\langle G\rangle$ provides the correct one-body limit as either particle's mass tends to infinity. The propagator also is invertible.

With regard to Z-graphs, one may compare the $++\rightarrow$ ++ piece of

$$
K_{\mathrm{ET}}^{(2)}\langle G\rangle K_{\mathrm{ET}}^{(2)}
$$

with the amplitude obtained at fourth order in the full $4 \mathrm{D}$ field theory. We find that the contribution of negativeenergy states agrees at leading order in $1 / m$ [18], and that this would be true even were different mass particles considered and the mass of either one taken to infinity. In other words, effects such as Fig. 11 are included in a bound-state calculation that employs Eq. (15). And this is true even if only the instantaneous ladder kernel $K_{\text {inst }}^{(2)}$ is used, because of our careful treatment of the one-body limit. 


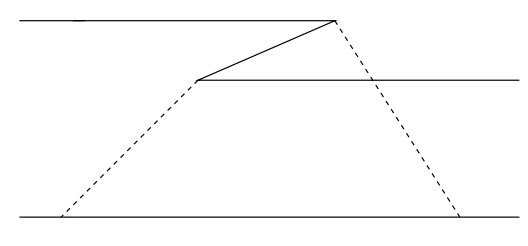

FIG. 1: One example of a Z-graph which is included in our $3 \mathrm{D}$ equation (15).

\section{THE INSTANTANEOUS $N N$ INTERACTION}

In Ref. [18] we examined the importance of retardation effects in $K_{\mathrm{ET}}$. We found that they had little impact on deuteron electromagnetic form factors. Hence in this work we report only on results obtained using Eq. (15) with an instantaneous interaction. This is consistent with use of the Bonn-B interaction that takes an instantaneous form in the center-of-mass frame. Other reductions to three dimensions are certainly possible but the result that retardation effects are small should not be sensitive to the reduction used.

As an example of how the reduction to an instantaneous, three-dimensional interaction is performed we consider the four-dimensional pseudovector pion kernel:

$$
K^{\pi}=\frac{g_{\pi}^{2} \tau_{1} \cdot \tau_{2}}{q^{2}-m_{\pi}^{2}} \frac{\gamma_{1}^{5} \gamma_{1} \cdot\left(p_{1}-p_{1}^{\prime}\right)}{2 m} \frac{\gamma_{2}^{5} \gamma_{2} \cdot\left(p_{2}-p_{2}^{\prime}\right)}{2 m}
$$

We use the identity:

$$
\begin{aligned}
& \bar{u}_{1}^{\prime} \gamma_{1}^{5} \frac{\gamma_{1} \cdot\left(p_{1}-p_{1}^{\prime}\right)}{2 m} u_{1}= \\
& \bar{u}_{1}^{\prime}\left\{\gamma_{1}^{5}+\frac{\left(p_{1}^{0}-p_{1}^{\prime 0}-\epsilon_{1}+\epsilon_{1}^{\prime}\right)}{2 m} \gamma_{1}^{5} \gamma_{1}^{0}\right\} u_{1},
\end{aligned}
$$

where $u_{1}^{\prime}$ and $u_{1}$ are Dirac spinors for positive-energy states, and carry out integrations over time-components of momenta as implied by the brackets in Eq. (17). To derive the instantaneous interaction we then take the static limit, i.e. assume $\left|E-\epsilon-\epsilon^{\prime}\right| \ll \omega$. In the language of effective field theory this means we consider only the effects of "potential pions" (which have $q_{0} \sim 0$ ) and ignore the impact of "radiation pions" $\left(q_{0} \sim m_{\pi}\right)$ on our results [39]. The result for the instantaneous ET interaction in ++ states is then:

$$
\begin{aligned}
K_{\mathrm{ET}}^{\pi} & =-\frac{g_{\pi}^{2}}{\mathbf{q}^{2}+m_{\pi}^{2}} \bar{u}_{1}^{\prime} \bar{u}_{2}^{\prime}\left\{\gamma_{1}^{5} \gamma_{2}^{5}-\frac{d_{1} d_{1}^{\prime}}{4 m^{2}} \gamma_{1}^{5} \gamma_{1}^{0} \gamma_{2}^{5} \gamma_{2}^{0}\right. \\
& \left.+\frac{d_{1}-d_{1}^{\prime}}{4 m}\left(\gamma_{1}^{5} \gamma_{1}^{0} \gamma_{2}^{5}+\gamma_{1}^{5} \gamma_{2}^{5} \gamma_{2}^{0}\right)\right\} u_{1} u_{2}
\end{aligned}
$$

where $u_{1}$ and $u_{2}$ are Dirac spinors, $d_{1}=E_{\mathbf{P}}-\epsilon_{1}\left(\mathbf{p}_{1}\right)-$ $\epsilon_{2}\left(\mathbf{p}_{2}\right)$ and $d_{1}^{\prime}=E_{\mathbf{P}}-\epsilon_{1}\left(\mathbf{p}_{1}^{\prime}\right)-\epsilon_{2}\left(\mathbf{p}_{2}^{\prime}\right)$. This interaction differs from a pseudoscalar one because of the terms involving $d_{1}$ and $d_{1}^{\prime}$, which are sub-leading in $1 / m$. They arise because - although we ignored retardation in the denominator of Eq. (22) - we must still choose an energy shell to determine the value of $p_{1}^{0}-p_{1}^{\prime}{ }^{0}$. The interaction (24) agrees with the $\tilde{\mu}=1$ interaction used by Adam, Göller, and Arenhövel [31], up to terms of order $p^{4} / m^{4}$.

\section{CURRENT CONSERVATION IN THE EQUAL-TIME APPROACH}

In order to compute deuteron electromagnetic form factors we must also construct a conserved electromagnetic deuteron current. In four dimensions there are two pieces to the impulse current, the first being determined by the $G_{0}$ part of the propagator:

$$
\begin{aligned}
& \bar{\Gamma}(P+q) G_{0}^{\prime} J_{0, \mu} G_{0} \Gamma(P)= \\
& \quad i \bar{\Gamma}(P+q) d_{1}\left(p_{1}\right) d_{2}\left(p_{2}+q\right) j_{\mu}^{(2)} d_{2}\left(p_{2}\right) \Gamma(P)+(1 \leftrightarrow 2),
\end{aligned}
$$

where $\Gamma$ is the solution of Eq. (10) for initial deuteron momentum $P=p_{1}+p_{2}, \Gamma^{\prime}$ is the solution for final deuteron momentum $P+q, d_{n}$ is the Dirac propagator for particle $n$, and $j_{\mu}^{(n)}$ is the usual one-nucleon current for particle $n$ :

$$
j_{\mu}^{(n)}=e^{(n)}\left[F_{1}^{(n)}\left(Q^{2}\right) \gamma_{\mu}^{(n)}+F_{2}^{(n)}\left(Q^{2}\right) \frac{i}{2 m} \sigma_{\mu \nu}^{(n)} q^{\nu}\right]
$$

where $e^{(n)}=\frac{|e|}{2}\left(1+\tau_{3}^{(n)}\right)$ is the charge. Using the WardTakahashi identity associated with formally modified but practically identical form of this current [40] it is easy to show that the current (25) is conserved, i.e.

$$
q^{\mu} \bar{\Gamma}(P+q) G_{0}^{\prime} J_{0, \mu} G_{0} \Gamma(P)=0,
$$

provided that $\Gamma$ obeys the ladder Bethe-Salpeter equation.

To obtain an equivalent three-dimensional current, we can replace $\Gamma \rightarrow \Gamma_{\mathrm{S}}$, yielding the conserved current

$$
\begin{aligned}
\mathcal{A}_{\mathrm{S}, \mu}=\bar{\Gamma}_{\mathrm{S}}^{\prime}\left\{\left\langle G_{0}^{\prime} J_{\mu} G_{0} K^{(2)} G_{0}\right\rangle+\right. & \left\langle G_{0}^{\prime} K^{(2) \prime} G_{0}^{\prime} J_{\mu} G_{0}\right\rangle \\
& \left.-\left\langle G_{0}^{\prime} J_{\mu} G_{0}\right\rangle\right\} \Gamma_{\mathrm{S}}(28)
\end{aligned}
$$

where $\Gamma^{\prime}, G_{0}^{\prime}$ and $K^{(2) \prime}$ include the momentum $Q$ from the photon absorption. Since we are considering the equal-time case we can also replace $K^{(2)} \rightarrow K_{\text {inst }}$, and because $K_{\text {inst }}$ has no dependence on time components of momenta, that replacement applied to Eq. (28) leads to

$$
\begin{aligned}
\mathcal{A}_{\mathrm{S}, \mu}=\bar{\Gamma}_{\mathrm{S}}^{\prime}\left\{\left\langle G_{0}^{\prime} J_{\mu} G_{0}\right\rangle K_{\mathrm{inst}}\left\langle G_{0}\right\rangle\right. & +\left\langle G_{0}^{\prime}\right\rangle K_{\mathrm{inst}}\left\langle G_{0}^{\prime} J_{\mu} G_{0}\right\rangle \\
& \left.-\left\langle G_{0}^{\prime} J_{\mu} G_{0}\right\rangle\right\} \Gamma_{\mathrm{S}}
\end{aligned}
$$

where the angle brackets indicate where integrations have been carried out over time components of momenta. This latter expression collapses to the impulse approximation form when Eq. (19) is used in the first two terms:

$$
\mathcal{A}_{\mathrm{S}, \mu}=\bar{\Gamma}_{\mathrm{S}}^{\prime}\left\langle G_{0}^{\prime} J_{\mu} G_{0}\right\rangle \Gamma_{\mathrm{S}} .
$$


The three terms in Eq. (28) are equal and they simplify to just one term in Eq. (30).

However, in this work we did not begin with the ladder BSE. Instead we began with the $4 \mathrm{D}$ equation (III). Constructing a conserved impulse approximation current for the vertex function which is the solution of Eq. (11) is a little more involved. In Ref. 18] we showed how to add a piece to the current (25) which results in a conserved current when $\Gamma$ is the solution of Eq. (11), giving a total $4 \mathrm{D}$ current

$$
\bar{\Gamma}(P+q) G_{0}^{\prime} J_{0, \mu} G_{0} \Gamma(P)+\bar{\Gamma}(P+q) G_{C \mu} \Gamma(P) .
$$

The explicit expression for $G_{C \mu}$ can be found in Ref. [18].

With this four-dimensional current in hand, we calculate the reduction to three dimensions as in Eq. (30). Replacing $\Gamma$ by $\Gamma_{\mathrm{ET}}$ we end up with

$$
\mathcal{A}_{\text {inst }, \mu}=\bar{\Gamma}_{\mathrm{ET}}^{\prime} \mathcal{G}_{\text {inst }, \mu}^{\gamma} \Gamma_{\mathrm{ET}}
$$

where:

$$
\mathcal{G}_{\text {inst }, \mu}^{\gamma}=\left\langle G^{\prime} J_{\mu} G\right\rangle=\left\langle G_{0}^{\prime} J_{\mu} G_{0}+G_{\mathrm{C} \mu}\right\rangle .
$$

This is analogous to the reduction employed for the bound-state equation itself. Once again, this reduction can be performed in a systematic fashion, but here we keep only the results for an instantaneous interaction. In that case $\mathcal{A}_{\text {inst }, \mu}$ is conserved, provided that $\Gamma_{\mathrm{ET}}$ is the solution of Eq. (15) using the instant form of the interaction. The explicit form of $\mathcal{G}_{\text {inst }}^{\gamma}$ is [18]:

$$
\begin{aligned}
\mathcal{G}_{\text {inst }, \mu}^{\gamma}\left(\mathbf{p}_{1}, \mathbf{p}_{2} ; P, Q\right)=i\left\langle d_{1}\left(p_{1}\right) d_{2}\left(p_{2}+Q\right) j_{\mu}^{(2)} d_{2}\left(p_{2}\right)\right\rangle \\
+i\left\langle d_{1}\left(p_{1}\right) d_{2}^{\tilde{c}}\left(p_{2}+Q\right) j_{c, \mu}^{(2)} d_{2}^{c}\left(p_{2}\right)\right\rangle+(1 \leftrightarrow 2)(34)
\end{aligned}
$$

Only the $\gamma^{\mu}$ piece of $j_{\mu}^{(n)}$ is relevant for charge conservation, since the piece proportional to $\sigma^{\mu \nu}$ is automatically conserved. Meanwhile, $d_{n}^{c}$ is a one-body Dirac propagator used in $G_{C}(P)$ to construct the approximation to the crossed-ladder graphs. Correspondingly, $d_{n}^{\tilde{c}}$ appears in $G_{C}(P+q)$. It does not equal $d_{n}^{c}$, even if particle $n$ is not the nucleon struck by the photon. Finally,

$$
j_{c, \mu}^{(2)}=\left(q_{2} \gamma_{\mu}^{(2)}-\tilde{j}_{\mu}^{(2)}\right) ; \quad \tilde{j}_{\mu}^{(2)}=q_{2} \frac{\hat{p}_{2 \mu}^{\prime}+\hat{p}_{2 \mu}}{\epsilon_{2}^{\prime}+\epsilon_{2}} \gamma_{0}^{(2)},
$$

with $\hat{p}_{2}=\left(\epsilon\left(\mathbf{p}_{2}\right), \mathbf{p}_{2}\right)$. The current defined by Eqs. (32)(35) includes the effects of photons creating Z-graphs by means of couplings from positive-energy states to negative-energy states.

This defines our impulse-approximation current. Detailed results for this current employed with the solutions of Eq. [15) were presented in Ref. [18]. The various refinements such as the $j_{c, \mu}^{(2)}$ term in Eq. (34) that were not considered by Hummel and Tjon [19] produce only very small changes for electron-deuteron scattering. They are incorporated in our calculations because they ensure current conservation when the ET propagator is used.

\section{DYNAMICAL BOOST}

To compute the current in Eq. (32) we consider electromagnetic matrix elements in the Breit frame where $\mathbf{P}=-\frac{1}{2} \mathbf{q}$ in the initial state and $\mathbf{P}^{\prime}=\frac{1}{2} \mathbf{q}$ in the final state. In the impulse approximation, using the instant interaction and the instant current in the Breit frame, the current matrix element may be expressed as

$$
\begin{array}{r}
\int \frac{d^{3} p}{(2 \pi)^{3}} \bar{\Gamma}\left(\mathbf{p}+\frac{1}{4} \mathbf{q} ; \frac{1}{2} \mathbf{q}\right) \mathcal{G}_{\text {inst }, \mu}^{\gamma}\left(\mathbf{p}-\frac{1}{2} \mathbf{q},-\mathbf{p} ; P, Q\right) \\
\Gamma\left(\mathbf{p}-\frac{1}{4} \mathbf{q} ;-\frac{1}{2} \mathbf{q}\right) .
\end{array}
$$

In the ETIA calculation of Ref. 18 Eq. (36) is calculated using vertex functions $\Gamma$ that are obtained by solving the bound-state equation

$$
\left(E_{\mathbf{P}}-\epsilon_{1}-\epsilon_{2}\right)\left|E_{\mathbf{P}}\right\rangle=v\left|E_{\mathbf{P}}\right\rangle,
$$

where $\epsilon_{n}=\sqrt{m^{2}+\mathbf{p}_{n}^{2}}$, for the state vector $\left|E_{\mathbf{P}}\right\rangle$, and then computing:

$$
\Gamma(\mathbf{k} ; \mathbf{P})=\left\langle\mathbf{k} ; \mathbf{P}\left|G_{0}^{-1}\right| E_{\mathbf{P}}\right\rangle .
$$

The operator

$$
H_{\mathrm{ET}}=\epsilon_{1}+\epsilon_{2}+v
$$

may be interpreted as the effective hamiltonian. With the usual conditions on the interaction $v$, it is bounded from below and may be used to generate a Hilbert space of states.

The interaction $v$ could be defined only in positiveenergy states, or it could be the effective interaction in positive-energy states that includes the effects of couplings to negative-energy states:,+--+ and -- . The couplings to negative-energy states obtained from the ET reduction do not produce any singularities in the effective interaction $v$. Once the interaction is defined in the c.m. frame of the two particles, where $\mathbf{P}=0$, the lowest eigenvalue is the rest mass of the two-particle bound state, $M$.

The problem here is that this does not necessarily guarantee that

$$
E_{\mathbf{P}}=\sqrt{M^{2}+\mathbf{P}^{2}}
$$

in other frames. That is because solving Eq. (37) in an arbitrary frame means using the ET interaction $K_{\mathrm{ET}}$ for $v$, and $K_{\mathrm{ET}}$ is calculated using Dirac spinors with arguments $\mathbf{p} \pm \frac{1}{2} \mathbf{P}$. Such an interaction depends on total momentum $\mathbf{P}$, and is not guaranteed to produce a bound state of the appropriate energy. In fact, in Ref. [18] we found that $K_{\mathrm{ET}}$ must be renormalized by a factor $\lambda\left(\mathbf{P}^{2}\right)$ if (40) is to hold in frames other than $\mathbf{P}=0$. The ad hoc factor $\lambda$ varies linearly from 1 at $\mathbf{P}^{2}=0$ to about 1.10 at $\mathbf{P}^{2}=4 \mathrm{GeV}^{2}[18$.

This occurs because the two-body ET interaction obtained by solving Eq. (37) using the ET interaction does 
not respect the Poincaré algebra. The basic requirement of Poincaré invariance is that states must transform under a unitary representation of the Poincaré group. The ten generators of translations in time, translations in space, boosts, and rotations are the hamiltonian operator, taken here to be of the form (39), the total momentum $\mathbf{P}$, the boost operator $\mathbf{K}$, and the angular momentum operator $\mathbf{J}$. They obey the following commutation relations.

$$
\begin{aligned}
{[\mathbf{P}, H]=0, } & {[\mathbf{J}, H]=0, } \\
{[\mathbf{K}, H]=i \mathbf{P}, } & {\left[K_{i}, P_{j}\right]=i \delta_{i j} H, } \\
{\left[J_{i}, P_{j}\right]=i \epsilon_{i j k} P_{k}, } & {\left[J_{i}, K_{j}\right]=i \epsilon_{i j k} K_{k}, } \\
{\left[J_{i}, J_{j}\right]=i \epsilon_{i j k} J_{k}, } & {\left[K_{i}, K_{j}\right]=-i \epsilon_{i j k} J_{k} . }
\end{aligned}
$$

The two-body boost problem is to constrain the interaction, $v$, such that the Poincaré generators satisfy the commutation rules that are required for Poincaré invariance. Our strategy will be to take $v$ to agree with $K_{\mathrm{ET}}$ in the two-body rest-frame $(\mathbf{P}=0)$ and then impose the conditions (41) in order to obtain approximate expressions for the matrix elements of $v$ in a frame where $\mathbf{P} \neq 0$. We denote the hamiltonian of this "approximate boost" procedure by $H_{\mathrm{ET}}$.

As mentioned earlier, we could instead employ an exact solution to the two-body boost problem by following Ref. 27] and introducing the interaction $K_{\mathrm{ET}}(\mathbf{P}=0)$ into the rest-frame mass operator $\hat{M}$. A hamiltonian that satisfies the Poincaré algebra would then be:

$$
H_{B T}=\sqrt{\hat{M}^{2}+\mathbf{P}^{2}} .
$$

The reason we choose not to apply Eq. (42) is that for electromagnetic interactions we also need current operators that are consistent with both the rest-frame and boosted hamiltonian. The ET formalism solves that problem by performing a consistent reduction of the quantum field theory. As discussed above, this yields currents which are consistent with $K_{\mathrm{ET}}$. Such a construction is significantly more difficult for $H_{B T}$. To indicate this we merely point out that if $H_{\mathrm{ET}}$ exactly satisfied the Poincaré algebra then the two different hamiltonians $H_{\mathrm{ET}}$ and $H_{B T}$ would be related by a unitary transformation, i.e., there would exist an operator $U$ with $U^{\dagger} U=1$ and:

$$
H_{\mathrm{ET}}=U H_{B T} U^{\dagger}
$$

If this $U$ were known, we could derive currents for use with states obtained from the hamiltonian (42) by transforming the currents derived in the ET formalism using the transformation (43). However, this unitary transformation is not known. So, in order to respect the consistency between the ET bound states and current operators, we will focus on the problem of finding an approximate boost that preserves the form of $H_{\mathrm{ET}}$ in frames with $\mathbf{P} \neq 0$.

It should be mentioned that if $\mathbf{P} \neq 0$ the hamiltonian $H_{\mathrm{ET}}$ has small differences from the operator $\epsilon_{1}+\epsilon_{2}+$
$K_{\mathrm{ET}}$ that was used in our analysis of the Ward-Takahashi identity. We leave the issue of boosting the currents so as to exactly maintain the Ward-Takahashi identity at $\mathbf{P} \neq 0$ as an unsolved problem.

An approximate solution to the boost problem within the subspace of an eigenvalue of the hamiltonian has been obtained in Ref. 26] for the case of two scalar particles. It provides an exact result for the energy of the two-body system. Here we review the basic elements of that proof and extend the result approximately to the case of two spin-half particles.

For the ET (and a number of other 3D) formalism(s), the two-body boost problem is the same as for the instant form of hamiltonian dynamics [4]. In this form of dynamics, total momentum and angular momentum operators do not depend on the interactions. They are:

$$
\begin{gathered}
\mathbf{P}=\mathbf{p}_{1}+\mathbf{p}_{2}, \\
\mathbf{J}=\mathbf{r}_{1} \times \mathbf{p}_{1}+\mathbf{r}_{2} \times \mathbf{p}_{2}+\frac{1}{2}\left(\sigma_{1}+\sigma_{2}\right) .
\end{gathered}
$$

It follows from $H$ commuting with $\mathbf{P}$ and $\mathbf{J}$ that $v$ must be translationally and rotationally invariant. The commutation relation of $\mathbf{K}$ and $H$ requires the boost operator to depend on the interaction. Such a boost is called "dynamical" in order to distinguish it from a "kinematical" boost for which the boost generator does not involve the interaction. The commutation rule between the dynamical boost generator and hamiltonian thus involves $v^{2}$ terms.

Bakamjian and Thomas 28] derived the form of the boost operator for instant dynamics. For a free boost, the operator is

$$
\mathbf{K}_{0}=\frac{1}{2}\left(\mathbf{r}_{1} \epsilon_{1}+\epsilon_{1} \mathbf{r}_{1}\right)-\frac{1}{2} \frac{\sigma_{1} \times \mathbf{p}_{1}}{\epsilon_{1}+m}+(1 \rightarrow 2) .
$$

When interactions are present, there is an interaction part of the hamiltonian, $v$, and an interaction part of the boost operator that is given approximately by

$$
\mathbf{K}_{v}=\frac{1}{2}(\mathbf{R} v+v \mathbf{R}),
$$

where $\mathbf{R}=\frac{1}{2}\left(\mathbf{r}_{1}+\mathbf{r}_{2}\right)$. This boost operator omits some terms from the standard Newton-Wigner construction [27] for $\mathbf{R}$, and likely could be improved. We leave such possible improvement of the boost for future analysis. Although it involves a significant approximation, in the case of the deuteron the boost operator of Eq. (47) yields the relationship (40) between the mass and energy of the bound state to good accuracy.

With the definitions given above, $\left[K_{i}, P_{j}\right]=i H \delta_{i j}$ is satisfied. However, there is an error term of order $1 / \mathrm{m}^{2}$ in the commutation relation $\left[K_{i}, K_{j}\right]=-i \epsilon_{i j k} J_{k}$. Accepting this error, the interaction $v$ must take an appropriate, but unknown, form consistent with $[\mathbf{K}, H]=i \mathbf{P}$.

Noting that the free-boost operator, $\mathbf{K}_{0}$, and the free hamiltonian, $H_{0}=\epsilon_{1}+\epsilon_{2}$, obey the commutation relation $\left[K_{0}, H_{0}\right]=i \mathbf{P}$, it follows that when interactiondependent terms are introduced into that commutation 
relation, their contributions must sum to zero, i.e.,

$$
\left[\mathbf{K}_{0}, v\right]+\left[\mathbf{K}_{v}, H_{0}\right]+\left[\mathbf{K}_{v}, v\right]=0 .
$$

This is equivalent to

$$
\frac{1}{2}\left[\mathbf{R}, H_{0} v+v H_{0}+v^{2}\right]+\frac{1}{4}[\mathbf{r} \Delta+\Delta \mathbf{r}, v]+\left[\mathbf{K}_{\sigma}, v\right]=0,
$$

where we have defined:

$$
\begin{aligned}
\mathbf{K}_{\sigma} & \equiv-\frac{1}{2} \frac{\sigma_{1} \times \mathbf{p}_{1}}{\epsilon_{1}+m}+(1 \rightarrow 2) \\
\Delta & \equiv \epsilon_{1}-\epsilon_{2} .
\end{aligned}
$$

Algebraic manipulations lead to

$$
\begin{array}{r}
{\left[\mathbf{R}, H_{0}\right] v+v\left[\mathbf{R}, H_{0}\right]+\frac{1}{2} H[\mathbf{R}, v]+\frac{1}{2}[\mathbf{R}, v] H} \\
+\frac{1}{2}(\Delta \mathbf{r} v-v \mathbf{r} \Delta)+\left[\mathbf{K}_{\sigma}, v\right]=0 .
\end{array}
$$

In quantum field theory the boost of a mass eigenstate appears to be kinematical. In fact the boost velocity $\beta=\mathbf{P} / E_{\mathbf{P}}$ depends upon the eigenvalue in the c.m. frame, $M$, which of course involves the interaction. This simple observation provides the key to solving the boost problem in instant dynamics. The $v^{2}$ terms in the dynamical boost may be eliminated when the boost is considered within the subspace of a single eigenvalue of the mass.

The boost velocity appropriate to a mass eigenstate does not enter the commutation relations. In order to restrict the boost to the subspace corresponding to a particular eigenvalue of $H$ we evaluate the matrix element of (51) between eigenstates of mass $M$ that obey Eq. (37). This yields:

$$
\begin{aligned}
\left\langle E_{\mathbf{P}}\right|\left(\left[\mathbf{R}, H_{0}\right] v\right. & +v\left[\mathbf{R}, H_{0}\right]+\frac{1}{2} E_{\mathbf{P}}[\mathbf{R}, v]+\frac{1}{2}[\mathbf{R}, v] E_{\mathbf{P}} \\
& \left.+\frac{1}{2}(\Delta \mathbf{r} v-v \mathbf{r} \Delta)+\left[\mathbf{K}_{\sigma}, v\right]\right)\left|E_{\mathbf{P}}\right\rangle=0(52)
\end{aligned}
$$

The $v^{2}$ pieces in the third and fourth terms of Eq. (51) have been eliminated in favor of the energy eigenvalue $E_{\mathbf{P}}$. Because of this, the boost appears to be kinematical in much the same way that the boost of a mass eigenstate in quantum field theory appears to be kinematical.

Performing the manipulations discussed in Ref. [26] we can convert Eq. (52) into an equation that is linear in $v$,

$$
\begin{aligned}
\left\langle E_{\mathbf{P}}\right|\left\{\frac{1}{2}\left(1+\frac{H_{0}}{E_{\mathbf{P}}}\right)\left[\mathbf{R}, H_{0}\right] v+\right. & \frac{1}{2} v\left[\mathbf{R}, H_{0}\right]\left(1+\frac{H_{0}}{E_{\mathbf{P}}}\right) \\
+\frac{1}{2} E_{\mathbf{P}}[\mathbf{R}, v]+\frac{1}{2}[\mathbf{R}, v] E_{\mathbf{P}} & +\frac{\mathbf{p} \cdot \mathbf{P}}{E_{\mathbf{P}}} \mathbf{r} v-v \mathbf{r} \frac{\mathbf{p} \cdot \mathbf{P}}{E_{\mathbf{P}}} \\
& \left.+\left[\mathbf{K}_{\sigma}, v\right]\right\}\left|E_{\mathbf{P}}\right\rangle=0 .(53)
\end{aligned}
$$

This equation is solved in momentum space in order to determine the form of $v$. Momentum-space matrix elements involve

$$
\begin{aligned}
\int \frac{d^{3} p^{\prime}}{(2 \pi)^{3}} \frac{d^{3} p}{(2 \pi)^{3}}\left\langle E_{\mathbf{P}} \mid \mathbf{p}^{\prime} ; \mathbf{P}\right\rangle E_{\mathbf{P}} \\
\left\{\left[\mathbf{A}\left(\mathbf{p}^{\prime} ; \mathbf{P}\right)+\mathbf{A}(\mathbf{p} ; \mathbf{P})+\mathbf{B}_{\mathrm{op}}\right]\left\langle\mathbf{p}^{\prime} ; \mathbf{P}|v| \mathbf{p} ; \mathbf{P}\right\rangle\right. \\
-i\left[\mathbf{K}_{\mathbf{P}, \sigma},\left\langle\mathbf{p}^{\prime} ; \mathbf{P}|v| \mathbf{p} ; \mathbf{P}\right\rangle\right]-i \mathbf{k}_{\sigma}^{\prime}\left\langle\mathbf{p}^{\prime} ; \mathbf{P}|v| \mathbf{p} ; \mathbf{P}\right\rangle \\
\left.+i\left\langle\mathbf{p}^{\prime} ; \mathbf{P}|v| \mathbf{p} ; \mathbf{P}\right\rangle \mathbf{k}_{\sigma}\right\}\left\langle\mathbf{p} ; \mathbf{P} \mid E_{\mathbf{P}}\right\rangle=0,(54)
\end{aligned}
$$

where

$$
\mathbf{A}(\mathbf{p} ; \mathbf{P})=\frac{1}{2 E_{\mathbf{P}}}\left(1+\frac{d(\mathbf{p} ; \mathbf{P})}{E_{\mathbf{P}}}\right) \frac{\partial d(\mathbf{p} ; \mathbf{P})}{\partial \mathbf{P}},
$$

with $d \equiv \epsilon_{1}(\mathbf{p} ; \mathbf{P})+\epsilon_{2}(\mathbf{p} ; \mathbf{P})$ being the value of $H_{0}$, and $A\left(\mathbf{p}^{\prime} ; \mathbf{P}\right)$ the same as $A(\mathbf{p} ; \mathbf{P})$, except with $\mathbf{p} \rightarrow \mathbf{p}^{\prime}$. Meanwhile,

$$
\begin{gathered}
\mathbf{B}_{\mathrm{op}} \equiv \frac{\partial}{\partial \mathbf{P}}+\frac{\mathbf{p}^{\prime} \cdot \mathbf{P}}{E_{\mathbf{P}}^{2}} \frac{\partial}{\partial \mathbf{p}^{\prime}}+\frac{\mathbf{p} \cdot \mathbf{P}}{E_{\mathbf{P}}^{2}} \frac{\partial}{\partial \mathbf{p}}, \\
\mathbf{K}_{\mathbf{P}, \sigma}=-\frac{1}{2} \frac{\left(\sigma_{1}+\sigma_{2}\right) \times \mathbf{P}}{E_{\mathbf{P}}\left(E_{\mathbf{P}}+M\right)},
\end{gathered}
$$

and

$$
\mathbf{k}_{\sigma}=-\frac{1}{2} \frac{\sigma_{1} \times \mathbf{q}_{1}}{E_{\mathbf{P}}\left(\epsilon_{1}+m\right)}+(1 \rightarrow 2)
$$

with

$$
\mathbf{q}_{1}=\mathbf{p}-\left(\frac{1}{2}-\frac{\epsilon_{1}+m}{E_{\mathbf{P}}+M}\right) \mathbf{P} .
$$

Derivatives in $\mathbf{B}_{\text {op }}$ act only on the interaction.

With spin effects omitted, a solution of Eq. (54) was obtained such that

$$
\left\langle\mathbf{p}^{\prime} ; \mathbf{P}|v| \mathbf{p} ; \mathbf{P}\right\rangle=f(\mathbf{p} ; \mathbf{P}) \tilde{v}\left(\mathbf{p}^{\prime}, \mathbf{p} ; \mathbf{P}\right) f\left(\mathbf{p}^{\prime} ; \mathbf{P}\right) .
$$

Here:

$$
\left(A(\mathbf{p} ; \mathbf{P})+\mathbf{B}_{\mathrm{op}}\right) f(\mathbf{p} ; \mathbf{P})=0 .
$$

The form of $\tilde{v}$ is deduced from the condition $\mathbf{B}_{o p} \tilde{v}=0$ and the boundary condition that, for $\mathbf{P}=0$, the interaction must be the c.m. frame one. Now constructing the rotational scalars

$$
\begin{aligned}
\mathbf{p}_{c}^{2} & =\mathbf{p}^{2}-\frac{(\mathbf{p} \cdot \mathbf{P})^{2}}{E_{\mathbf{P}}^{2}} \\
\mathbf{p}_{c}^{\prime 2} & =\mathbf{p}^{\prime 2}-\frac{\left(\mathbf{p}^{\prime} \cdot \mathbf{P}\right)^{2}}{E_{\mathbf{P}}^{2}}, \\
\mathbf{p}_{c} \cdot \mathbf{p}_{c}^{\prime} & =\mathbf{p} \cdot \mathbf{p}-\frac{(\mathbf{p} \cdot \mathbf{P})\left(\mathbf{p}^{\prime} \cdot \mathbf{P}\right)}{E_{\mathbf{P}}^{2}}
\end{aligned}
$$

one can check that

$$
\begin{aligned}
\mathbf{B}_{o p} \mathbf{p}_{c}^{2} & =0, \\
\mathbf{B}_{o p} \mathbf{p}_{c}^{2} & =0 \\
\mathbf{B}_{o p} \mathbf{p}_{c} \cdot \mathbf{p}_{c}^{\prime} & =0 .
\end{aligned}
$$


So, it follows that if $\tilde{v}=v_{c}\left(\mathbf{p}_{c}^{\prime}, \mathbf{p}_{c}\right)$ is an arbitrary function of $\mathbf{p}_{c}^{2}, \mathbf{p}_{c}^{\prime 2}$ and $\mathbf{p}_{c} \cdot \mathbf{p}_{c}^{\prime}$, then

$$
\mathbf{B}_{o p} v_{c}\left(\mathbf{p}_{c}^{\prime}, \mathbf{p}_{c}\right)=0
$$

and the condition $\mathbf{B}_{o p} \tilde{v}=0$ is satisfied. Thus, in the absence of spin,

$$
\tilde{v}\left(\mathbf{p}^{\prime}, \mathbf{p} ; \mathbf{P}\right)=v_{c}\left(\mathbf{p}_{c}^{\prime}, \mathbf{p}_{c}\right) .
$$

where

$$
\mathbf{p}_{c} \equiv \mathbf{p}-\frac{(\mathbf{p} \cdot \mathbf{P}) \mathbf{P}}{E_{\mathbf{P}}\left(E_{\mathbf{P}}+M\right)},
$$

and

$$
\mathbf{p}_{c}^{\prime} \equiv \mathbf{p}^{\prime}-\frac{\left(\mathbf{p}^{\prime} \cdot \mathbf{P}\right) \mathbf{P}}{E_{\mathbf{P}}\left(E_{\mathbf{P}}+M\right)} .
$$

In the c.m. frame, $\mathbf{p}_{c}$ and $\mathbf{p}_{c}^{\prime}$ are the standard relative momenta. When the total momentum is in the $z$ direction, the $z$-component of relative momentum $\mathbf{p}_{c}$ is contracted according to $p_{c z}=p_{z} / \gamma$, where $\gamma=E_{\mathbf{P}} / M$. The components of relative momenta perpendicular to the total momentum are unaffected: $\mathbf{p}_{c \perp}=\mathbf{p}_{\perp}$. The same rule applies to $\mathbf{p}_{c}^{\prime}$.

Solving Eq. 61) for $f(\mathbf{p} ; \mathbf{P})$ subject to the boundary condition that $f(\mathbf{p}, 0)=1$ we find:

$$
f^{2}(\mathbf{p} ; \mathbf{P})=\frac{M}{E_{\mathbf{P}}}\left(\frac{E_{\mathbf{P}}-\epsilon_{1}(\mathbf{p} ; \mathbf{P})-\epsilon_{2}(\mathbf{p} ; \mathbf{P})}{M-2 \epsilon\left(\mathbf{p}_{c} ; 0\right)}\right) .
$$

This completes the proof that the commutator relation $[\mathbf{K}, H]=i \mathbf{P}$ is satisfied exactly in the subspace of eigenvalue $E_{\mathbf{P}}$ when spin effects are omitted.

When spin effects are included, we find that if the c.m. frame interaction is expressed in terms of a matrix element involving Dirac spinors depending upon $\mathbf{p}_{c}$ and $\mathbf{p}_{c}^{\prime}$, then the commutator term involving $\mathbf{K}_{\mathbf{P}, \sigma}$ and the spin terms generated by $\mathbf{B}_{o p}$ acting on the Dirac spinors in Eq. (54) cancel. That is, let

$$
v_{c}\left(\mathbf{p}_{c}^{\prime}, \mathbf{p}_{c}\right)=\bar{u}_{1}\left(\mathbf{p}_{c}^{\prime}\right) \bar{u}_{2}\left(\mathbf{p}_{c}^{\prime}\right) \hat{v}\left(\mathbf{p}_{c}^{\prime}, \mathbf{p}_{c}\right) u_{1}\left(\mathbf{p}_{c}\right) u_{2}\left(\mathbf{p}_{c}\right)
$$

where $\hat{v}\left(\mathbf{p}_{c}^{\prime}, \mathbf{p}_{c}\right)$ is a rotationally invariant function of its arguments that may involve local Dirac matrix structures, for example the Fermi covariants. The Dirac spinors $u_{1}, u_{2}$, etc. in (69) omit the usual Pauli spinor factors, i.e., they are boost operators for Dirac spinors similar in form to Eq. (83) below. It follows that the spinor matrix element in (69) involves products of terms like $\sigma_{1} \cdot \mathbf{p}_{c}$ and $\sigma_{2} \cdot \mathbf{p}_{c}$ from the spinors and $\hat{v}$. Now consider the Dirac matrix structure $\Gamma_{1} \Gamma_{2}$ that arises from these products. The cancellation we want will occur, provided that

$$
\mathbf{B}_{\mathrm{op}} \Gamma_{1} \Gamma_{2}-i\left[\mathbf{K}_{\mathbf{P} \sigma}, \Gamma_{1} \Gamma_{2}\right]=0 .
$$

But the Dirac structures $\Gamma_{1} \Gamma_{2}$ either involve $\sigma_{1} \cdot \sigma_{2}$ oras in the case of $\pi N N$ coupling-factors of $\sigma_{n} \cdot \mathbf{p}_{c}$. A straightforward calculation then shows that,

$$
\mathbf{B}_{o p} \sigma_{1} \cdot \mathbf{p}_{c}-i\left[\mathbf{K}_{\mathbf{P}, \sigma}, \sigma_{1} \cdot \mathbf{p}_{c}\right]=0,
$$

and that a similar identity holds also for argument $\mathbf{p}_{c}^{\prime}$ and spin operator $\sigma_{2}$. Since $\mathbf{K}_{\mathbf{P}, \sigma}$ commutes with $\sigma_{1} \cdot \sigma_{2}$, it follows that:

$$
\mathbf{B}_{o p} v_{c}\left(\mathbf{p}_{c}^{\prime}, \mathbf{p}_{c}\right)-i\left[\mathbf{K}_{\mathbf{P}, \sigma}, v_{c}\left(\mathbf{p}_{c}^{\prime}, \mathbf{p}_{c}\right)\right]=0 .
$$

The remainder of the boost operator can be understood as requiring a rotation of spins when $\mathbf{P} \neq 0$,

$$
R_{i}(\mathbf{p}) \approx 1+\frac{i \mathbf{P} \cdot \sigma_{i} \times \mathbf{p}}{2\left(\epsilon_{c}+m\right)^{2}}+\cdots,
$$

where $i=1$ or 2 and omitted terms are of order $1 / m^{4}$. 42 That is, the interaction in a frame with nonzero total momentum should have the final form

$$
\begin{array}{r}
\left\langle\mathbf{p}^{\prime} ; \mathbf{P}|v| \mathbf{p} ; \mathbf{P}\right\rangle=f\left(\mathbf{p}^{\prime} ; \mathbf{P}\right) R_{1}^{\prime} R_{2}^{\prime} \bar{u}_{1}\left(\mathbf{p}_{c}^{\prime}\right) \bar{u}_{2}\left(\mathbf{p}_{c}^{\prime}\right) \\
\hat{v}\left(\mathbf{p}_{c}^{\prime}, \mathbf{p}_{c}\right) u_{1}\left(\mathbf{p}_{c}\right) u_{2}\left(\mathbf{p}_{c}\right) R_{1} R_{2} f(\mathbf{p} ; \mathbf{P}) .
\end{array}
$$

Factors $R_{i}^{\prime}$ and $R_{i}$ allow $1 / \mathrm{m}^{2}$ parts of the terms involving $\mathbf{k}_{\sigma}^{\prime}$ and $\mathbf{k}_{\sigma}$ to be canceled by new terms generated when $\mathbf{B}_{o p}$ acts on the spin rotation factors in Eq. (54). However, there are order $1 / \mathrm{m}^{4}$ errors that cannot be canceled: we accept these as error terms of the boost related to the fact that our boost generator is not exact.

In summary, we find that if an arbitrary c.m. frame interaction that is rotationally and translationally invariant is constructed in other frames according to Eq. (74), then a bound state of mass $M$ defined by solution of the c.m. frame equation,

$$
\left[M-2 \epsilon\left(\mathbf{p}^{\prime} ; 0\right)\right] \Psi_{c}\left(\mathbf{p}^{\prime}\right)=\int \frac{d^{3} p}{(2 \pi)^{3}} v_{c}\left(\mathbf{p}^{\prime}, \mathbf{p}\right) \Psi_{c}(\mathbf{p}),
$$

corresponds in another frame to a state of energy $E_{\mathbf{P}}$, given by solving the eigenvalue equation:

$$
\begin{array}{r}
{\left[E_{\mathbf{P}}-\epsilon_{1}\left(\mathbf{p}^{\prime} ; \mathbf{P}\right)-\epsilon_{2}\left(\mathbf{p}^{\prime} ; \mathbf{P}\right)\right] \Psi\left(\mathbf{p}^{\prime} ; \mathbf{P}\right)=} \\
\int \frac{d^{3} p}{(2 \pi)^{3}}\left\langle\mathbf{p}^{\prime}, \mathbf{P}|v| \mathbf{p} ; \mathbf{P}\right\rangle \Psi(\mathbf{p} ; \mathbf{P}) .
\end{array}
$$

Wave functions for the initial and final state are now obtained from Eq. (76) using the interaction defined as in Eq. (74). This provides wave functions of energy $E_{\mathbf{P}}$ that correspond to a mass $M$ in the c.m. frame of the twonucleons, and which can be inserted into Eq. (36). The spin rotation factors are given approximately by Eq. (73), within errors of order $1 / m^{4}$. We have omitted spin rotation effects in our calculations but we find the correct relation between energy and mass with high accuracy. The boost rule just stated is approximate because we have omitted terms that would be required in order to achieve an exact solution of the Poincaré algebra. It is designed to be consistent with the currents derived using the ET reduction.

\section{PSEUDOVECTOR-PION CONTRIBUTION TO THE ISOSCALAR TWO-BODY FOUR CURRENT}

The derivation presented in Sec. IV involves a straightforward reduction of conserved four-dimensional matrix 
elements of the electromagnetic current. It neglects retardation effects (known to be small) but also neglects the dependence on the relative energy $p_{0}$ of meson-nucleon vertex functions.

As we will show here, keeping the $p_{0}$-dependence of the $\pi N N$ vertex results in an additional contribution to the $3 \mathrm{D}$ current that can be interpreted as a "mesonexchange current" contribution to the current operator. As stressed by Friar [30] and Adam and Arenhövel [43], it is critical that these MEC contributions be evaluated in a manner consistent with that used to evaluate the $N N$ interaction. Here we implement this consistency by recalling that the instant $N N$ potential $K_{\mathrm{ET}}$ is derived by computing the ET matrix elements using a four-dimensional kernel and then taking the static limit $\left|E-\epsilon-\epsilon^{\prime}\right| \ll \omega$ in the denominator. Thus in order to obtain a consistent $\mathcal{A}_{\mu}$ for pseudovector pion coupling we should implement Eq. (29) with the $4 \mathrm{D} K$ given by Eq. (22) and also take the static limit there. This leads to a pion-range contribution to the isoscalar four current, one first identified by Riska a number of years ago. This contribution was not included in calculations of Ref. [18] and its omission is part of the reason that the data for $A$ is underpredicted there. It is depicted on the right-hand side of the upper line of Fig. 2] For pseudovector $\pi N$ coupling, the extra current is derived from Eq. (28) by evaluating the expressions using the positive-energy part of each Dirac propagator and the $\pi$-exchange kernel of Eq. (22). Following the procedure outlined above Eq. (24) leads to an expression very similar to Eq. (29), as follows

$$
\begin{gathered}
\mathcal{A}_{\mu}=\bar{\Gamma}_{\mathrm{ET}}^{\prime}\left\{\left\langle G_{0}^{\prime} J_{\mu} G_{0}\right\rangle K_{\mathrm{ET}}^{\mathrm{A}, \pi}\left\langle G_{0}\right\rangle+\left\langle G_{0}^{\prime}\right\rangle K_{\mathrm{ET}}^{\mathrm{B}, \pi}\left\langle G_{0}^{\prime} J_{\mu} G_{0}\right\rangle\right. \\
\left.-\left\langle G_{0}^{\prime} J_{\mu} G_{0}\right\rangle\right\} \Gamma_{\mathrm{ET}} .
\end{gathered}
$$

where

$$
\begin{aligned}
K_{\mathrm{ET}}^{\mathrm{A}, \pi}= & K_{\mathrm{ET}}^{\mathrm{B}, \pi}= \\
- & \frac{g_{\pi}^{2} \tau_{1} \cdot \tau_{2}}{\mathbf{q}^{2}+m_{\pi}^{2}} \bar{u}_{1}^{\prime} \bar{u}_{2}^{\prime}\left\{\gamma_{1}^{5}+\frac{-r_{A}-\epsilon_{1}+\epsilon_{1}^{\prime}}{2 m} \gamma_{1}^{5} \gamma_{1}^{0}\right\} \\
& \left\{\gamma_{2}^{5}+\frac{r_{A}-\epsilon_{2}+\epsilon_{2}^{\prime}}{2 m} \gamma_{2}^{5} \gamma_{2}^{0}\right\} u_{1} u_{2},
\end{aligned}
$$

with $r_{A}=\frac{1}{2}\left(E_{\mathbf{P}}-2 \epsilon_{2}+\epsilon_{2}^{\prime}-\epsilon_{1}^{\prime}\right)$. The factors in curly brackets in Eq. (78) are similar to those in Eq. (24). The replacement of $p_{1}^{0}-p_{1}^{\prime 0}$ by $-r_{A}$ occurs as the result of performing the integrations over time-components of momenta as dictated by the reduction to three dimensions. Conversely $p_{2}^{0}-p_{2}^{\prime 0}$ gets replaced by $+r_{A}$.

The extra terms that arise due to the retention of $p_{0}^{\prime}-p_{0}$ pieces in the numerator of the pion exchange can now be determined by comparing Eq. (77) with Eq. (29). This leads to the following expression, which is what we calculate below in order to include the PV-contact MEC:

$$
\begin{aligned}
& \mathcal{A}_{\mathrm{MEC}, \mu}=\bar{\Gamma}_{\mathrm{ET}}^{\prime}\left\{\left\langle G_{0}^{\prime} J_{\mu} G_{0}\right\rangle\right. \\
& +\left\langle G_{0}^{\prime} J_{\mu} G_{0}\right\rangle\left(K_{\mathrm{ET}}^{\mathrm{A}, \pi}-K_{\mathrm{ET}}^{\pi}\right)\left\langle G_{0}\right\rangle \\
& \left.+\left\langle G_{0}^{\prime}\right\rangle\left(K_{\mathrm{ET}}^{\mathrm{B}, \pi}-K_{\mathrm{ET}}^{\pi}\right)\left\langle G_{0}^{\prime} J_{\mu} G_{0}\right\rangle\right\} \Gamma_{\mathrm{ET}} .
\end{aligned}
$$
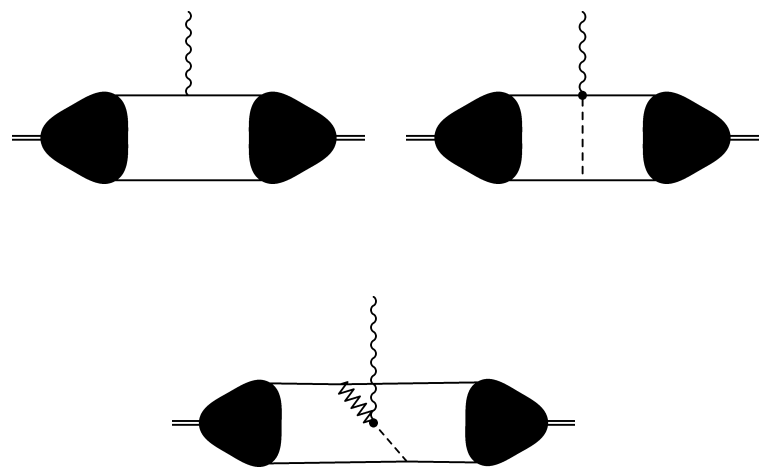

FIG. 2: On the left of the upper line we depict the positiveenergy-state impulse-approximation mechanism for electrondeuteron scattering. On the right of that line is the PVcontact MEC. In the bottom line we show the $\rho \pi \gamma$ MEC, with the $\rho$-meson represented by the wavy line. In each case the blobs are the deuteron vertex functions $\Gamma$.

In fact, the dominant contribution here is due to $J_{0}$. Contributions to $\vec{J}$ are suppressed by $p / M$ and are numerically small, but they are included automatically in our formalism. The resultant expression for the two-body piece of $J_{0}$ agrees with Adam et al. 31] at leading order in $p / m$. We have also checked that the expression (79) for this PV-coupling current agrees numerically with the more conventional expression as a two-body operatoragain, to leading order in $p / m$.

\section{THE $\rho \pi \gamma$ EXCHANGE CURRENT}

Because of the quantum numbers of the deuteron, the $\rho \pi \gamma$ meson-exchange current is generally thought to be the lowest-mass mesonic excitation that makes a contribution to the electromagnetic deuteron current. The $\rho \pi \gamma$ exchange current can be used to provide a resonancesaturation model of the two-body contribution to $\vec{J}$. This meson-exchange piece of $G_{M}$ is of $O\left(e \mathcal{P}^{4}\right)$, and has an unknown coupling from $\mathcal{L}_{\pi N}^{(3)}$. The value of this unknown coupling can be modeled by assuming it is dominated by the $\rho$ excitation, see e.g. Ref. [44].

The Lagrangian governing the $\rho \pi \gamma$ vertex is:

$$
\mathcal{L}=-e \frac{g_{\rho \pi \gamma}}{2 m_{\rho}} \epsilon_{\alpha \beta \gamma \delta} F^{\alpha \beta} \vec{\rho}^{\gamma} \cdot \partial^{\delta} \vec{\pi}
$$

while the $\rho N N$ vertex has the form

$$
\mathcal{L}_{\rho N N}=\bar{N} g_{\rho}\left(\gamma^{\mu}+i \frac{f_{\rho}}{g_{\rho}} \frac{\sigma^{\mu \nu} q_{\nu}}{2 m}\right) N .
$$

This yields the two-body current depicted on the lower line of Fig. 2] Note that this current is automatically conserved. In calculating it we use the same vertex functions and form factors employed at the $\pi N$ and $\rho N$ vertices in calculating the $N N$ potential. The coupling $g_{\rho \pi \gamma}=0.563$ 
is extracted from the decay $\rho \rightarrow \pi \gamma$. This value was used by Hummel and Tjon 19], however, Truhlík, Smejkal and Khanna 45] recently have calculated somewhat higher values, namely .585 ( based on $\rho^{ \pm}$decay) and .610 ( based on $\rho^{0}$ decay). Although the overall sign of $g_{\rho \pi \gamma}$ is not determined by the experimental data, a recent lattice QCD calculation gives a positive value [46]. Quark models such as the one of Ref. 47] also provide a positive value for $g_{\rho \pi \gamma}$. We have used $g_{\rho \pi \gamma}=.563$ in our calculations. For consistency with the Bonn-B potential the ratio $f_{\rho} / g_{\rho}$ was initially chosen to be 6.1 . However, $f_{\rho} / g_{\rho}$ is not well determined by fitting the $N N$ data and recent work has suggested that a smaller value $f_{\rho} / g_{\rho} \approx 4.5$ is acceptable [4]. This would be closer to the $f_{\rho} / g_{\rho} \approx 3.7$ value obtained when vector-meson dominance is used to explain the isovector anomalous magnetic moment of the nucleon [49]. We note that in nonrelativistic calculations the $f_{\rho} / g_{\rho}$ term often is neglected altogether on the grounds that it is higher order in $1 / m$ [8]. In contrast, Hummel and Tjon performed relativistic calculations of deuteron form factors using $f_{\rho} / g_{\rho}=6.8$. They found that the contribution of the tensor $\rho N$ coupling to $G_{M}$ cancels with the contribution of the vector $\rho N$ coupling when $|\mathbf{q}|$ is about $1 \mathrm{GeV}$. This throws into question the validity of neglecting the tensor piece of the $\rho N N$ coupling if $f_{\rho} / g_{\rho}$ is as large as this.

Even were $f_{\rho} / g_{\rho}$ known precisely there is still another significant source of uncertainty in evaluating this exchange current: its contribution to electron-deuteron scattering depends crucially on the behavior of the current operator as a function of $Q^{2}$, and hence on the $\rho \pi \gamma$ form factor. In the work of Hummel and Tjon vectormeson dominance was used to obtain a $\rho \pi \gamma$ form factor given solely by the $\omega$ meson:

$$
F_{\rho \pi \gamma}\left(Q^{2}\right)=\frac{1}{Q^{2}+m_{\omega}^{2}} .
$$

This same $\rho \pi \gamma$ form factor is also employed in the nonrelativistic calculations of Refs. [8, 9]. Other calculations have used form factors based on quark models [20, 47]. Such form factors tend to reduce the contribution of this MEC, which is also very sensitive to the cutoff masses in the $\pi N$ and $\rho N$ vertices.

No experimental data are available to constrain the form factor that should be used for the $\rho \pi \gamma$ vertex. Lattice QCD calculations can determine the form factor for on-shell $\pi$ and $\rho$ mesons. Recent lattice calculations of Edwards [46] indicate that for $Q^{2}$ up to $0.6 \mathrm{GeV}^{2}$ the $\rho \pi \gamma$ for factor agrees with the vector-meson dominance form (82).

\section{Z-GRAPHS}

In the full ET analysis the interactions and propagators are defined on a complete set of Dirac plane-wave states:,,+++--+ and -- . The bound-state equation is solved with all possible couplings between these states, and the current matrix elements allow for all possible transitions between the states. Physically, coupling to negative-energy states allows positive-energy, planewave solutions of the free Dirac equation to be distorted in the presence of a meson field. The simplest example of this effect - and one of the motivations for including itis the shift of the mass of a Dirac particle in a uniform external scalar field: $m \rightarrow m^{*}=m+S$. Positive-energy solutions of the Dirac equation in this scalar field have the form

$$
u(p) \approx\left(\begin{array}{c}
1 \\
\frac{\sigma \cdot \mathbf{p}}{2 m^{*}}
\end{array}\right) .
$$

To recapture the effect of the replacement $m \rightarrow m^{*}$ orderby-order in $S$ the coupling of positive-energy states of mass $m$ to negative-energy states must be included in the calculation. So allowing for couplings between all positive- and negative-energy components - as we do in the full ET formalism - is a general way to incorporate this kind of distortion of the Dirac spinors in the theory.

It is interesting to explore the extent to which these effects of coupling to negative-energy states are captured if we include them only via first-order perturbation theory in the nucleon-nucleon potential. Thus, instead of solving (15) in all $\rho$-spin sectors, $\rho_{1}, \rho_{2}=++,+-,-+,--$, we solve it in ++ states alone to obtain $\Gamma_{\mathrm{ET}}^{++}$and then generate the couplings to negative-energy states by treating the difference between the full interaction and the ++ states-only interaction in first-order perturbation theory. This leads to the following expression for the current matrix element:

$$
\begin{aligned}
\mathcal{A}_{\mathrm{Z}, \mu}= & \bar{\Gamma}_{\mathrm{ET}}^{\prime++}\left\{\left\langle G_{0}^{\prime} J_{\mu} G_{0}\right\rangle\right. \\
& +\left\langle G_{0}^{\prime} J_{\mu} G_{0}\right\rangle\left(K_{\mathrm{ET}}-K_{\mathrm{ET}}^{++,++}\right)\left\langle G_{0}\right\rangle \\
+ & \left.\left\langle G_{0}^{\prime}\right\rangle\left(K_{\mathrm{ET}}-K_{\mathrm{ET}}^{++,++}\right)\left\langle G_{0}^{\prime} J_{\mu} G_{0}\right\rangle\right\} \Gamma_{\mathrm{ET}}^{++} .(84)
\end{aligned}
$$

The second and third terms in Eq. (84) provide the leading-order corrections due to negative-energy components in the initial and final states, respectively. The leading-order Z-graph calculations we present below are performed in this way.

\section{RESULTS}

With all the theoretical pieces of the puzzle assembled we now calculate electron-deuteron scattering observables. The vertex functions employed are the ones calculated with all positive and negative-energy states included, as described in Ref. [18]. If the negative-energy states are dropped the interaction is exactly the Bonn-B potential for the Thompson equation, as derived and fitted to $N N$ phase shifts in Ref. 25]. This model gives a reasonable fit to the $N N$ data, and good deuteron static properties, although it is not as good a fit as some more recent $N N$ potentials $[8,50,51]$. When negative-energy 
states are included the deuteron binding energy changes slightly. To compensate for this we adjust the $\sigma$-meson coupling from the value of the fit in Ref. [25], $\frac{g_{\sigma}^{2}}{4 \pi}=8.08$, to $\frac{g_{\sigma}^{2}}{4 \pi}=8.55$.

The single-nucleon form factors are taken from the recent work of Kelly 52 that includes data from Jefferson Laboratory on the ratio $G_{E_{p}} / G_{M_{p}}$ and $G_{E_{n}}$. However, we find that the results are very close to those obtained using the nucleon form factors of Mergell et al. [53]. Both parameterizations incorporate constraints on the asymptotic shape of $F_{1}$ and $F_{2}$ using arguments from perturbative QCD.

We see clearly in Fig. 3 that the baseline ETIA calculation underpredicts the $A$ data for $Q^{2}=25-75 \mathrm{fm}^{-2}$, i.e. $Q^{2}=1-3 \mathrm{GeV}^{2}$. This is true for both the curve labeled $\mathrm{ET}(++)$ - which includes only ++ states - and the one labeled ET(neg) - which includes the effects of all positive- and negative-energy states to all orders in $K_{\mathrm{ET}}$. Data from the two JLab experiments that have measured $A$ [3, 4] are denoted by triangles and squares. Note that these experiments confirm the trend of the SLAC data of Arnold et al. The extant experimental data for $B$ [55, 56, 57] (which, as yet, include no JLab data) are even less well-described. Already at $Q^{2} \lesssim 25$ $\mathrm{fm}^{-2} \approx 1 \mathrm{GeV}^{2}$ there is significant disagreement between our ETIA calculation and the data.

In Fig. [3 we also present results for observables $A$ and $B$ showing the effects of the approximate dynamical boost. The curves are labeled Boost $(++)$ and Boost(neg). The net effect of the dynamical boost is significant but not large. For either the ++ calculation or the one including all states, the boost shifts the minimum in the calculated $B$ observable to somewhat higher $Q^{2}$. As seen in Fig. 31 the $A$ observable is not much altered by the boost. These results suggest that further refinement of the approximate boost developed here, while theoretically interesting, would have little phenomenological impact on these observables.

A similar result is seen for observable $t_{20}$ in Fig. 4 Although effects on observables are not large, inclusion of the approximate dynamical boost in our calculations is an important step forward because it ensures that the deuteron mass is independent of frame, whereas an ad hoc factor $\lambda\left(\mathbf{P}^{2}\right)$ in $K_{\mathrm{ET}}$ was needed for this purpose in prior work.

Calculations with and without the PV-coupling MEC are shown in Fig. [5 for $A$ and $B$ and in Fig. 6 for $t_{20}$. This MEC, which must be included because we chose to employ pseudovector $\pi N$ coupling, provides a significant increase in the $A$ observable, small changes in $B$ and a significant shift of $t_{20}$ toward smaller $Q$. The increase in $A$ helps to move the result into better agreement with the experimental data. But our results for $B$ still decrease too fast with $Q$ and the minimum occurs at too low a value of $Q$. The shift of $t_{20}$ aligns our results quite well with the JLab data of Abbott et al. [1, 2].

In Fig. 7 we present our results for $A$ and $B$ when the

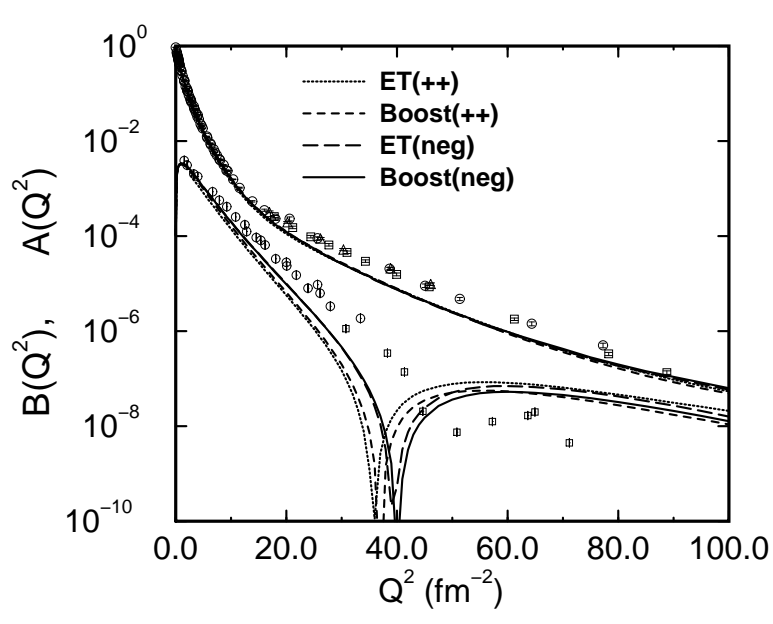

FIG. 3: The deuteron structure functions $A$ and $B$. The experimental data for $A$ of Refs. [54, 55] are denoted by the open circles, while those of Refs. [2, 3, 4] are represented by triangles and squares. The experimental data for $B$ of Refs. [55, 56] are shown by open circles, while those of Ref. 57] are shown by squares. The dotted and short-dashed lines labeled $\mathrm{ET}(++)$ and $\mathrm{ET}(\mathrm{neg})$ are based on the ET calculation without the dynamical boost. The long-dashed and solid curves labeled Boost(++) and Boost(neg) include the dynamical boost.

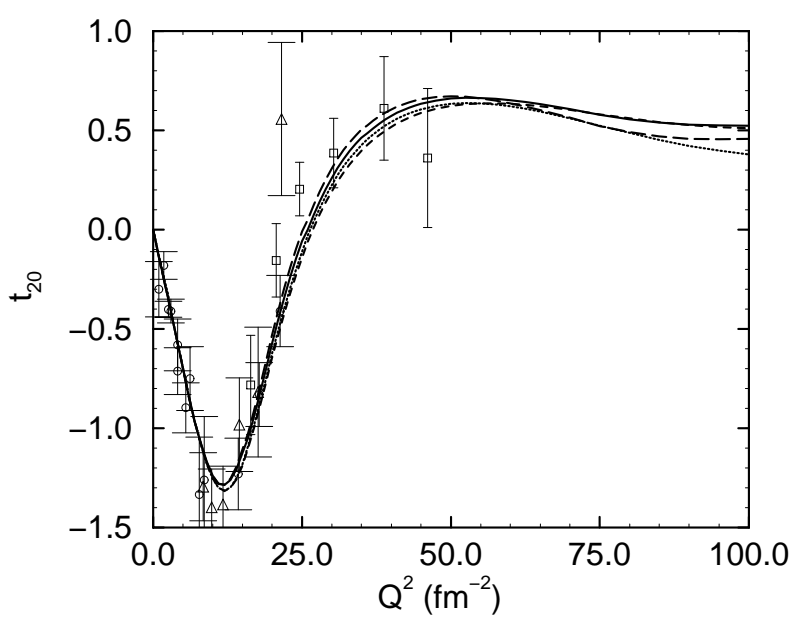

FIG. 4: The tensor-polarization observable $t_{20}$. The older experimental data 58] and the NIKHEF data of Bouwhuis et al. [59] are shown by circles, the JLab data of Abbott et al. 1] by squares and the Novosibirsk data of Nikolenko et al. 60. by triangles. The lines have the same meaning as in Fig. 3

$\rho \pi \gamma$ MEC is included using the vector-meson-dominance form factor (82) as in the work of Hummel and Tjon. Results are shown for three values of the tensor coupling ratio $f_{\rho} / g_{\rho}=0,3$, and 6.1 and all calculations include the full set of positive- and negative-energy states and the PV-coupling MEC. At larger values of $Q$, the calculated 


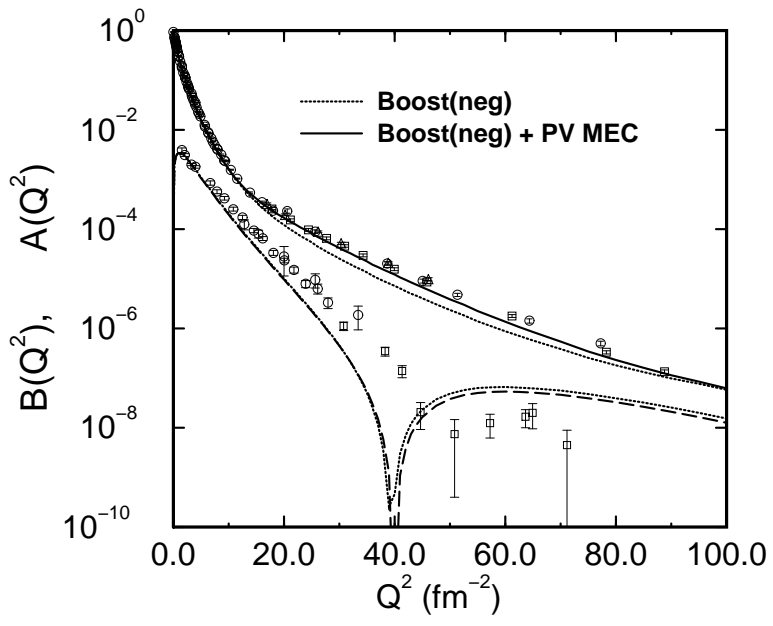

FIG. 5: The deuteron structure functions $A$ and $B$ with and without the PV-coupling MEC. Experimental data points are as described in caption to Figure 3

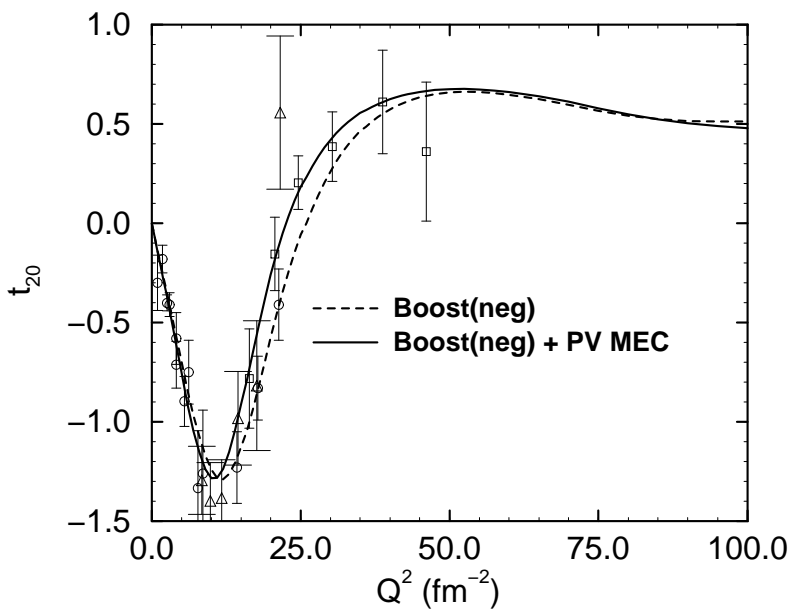

FIG. 6: The tensor-polarization observable $t_{20}$ with and without the PV-coupling MEC. Both calculations include all positive- and negative-energy sectors. Experimental data points are as described in caption of Figure 4

values of $A$ are increased by the $\rho \pi \gamma$ MEC. For the ratio $f / g=0$, the $\rho \pi \gamma$ contribution provides good agreement with the data for observable $A$.

Meanwhile, Fig. 8 shows results for the tensor observable $t_{20}$, which is rather insensitive to the $\rho \pi \gamma$ contribution. In contrast, this exchange current has a significant impact on the observable $B$. In particular, different choices for the tensor $\rho N$ coupling change the results markedly, as is evident in the lower curves of Fig. 7 It is clear from these results that the minimum of $B$ is especially sensitive to the value of $f_{\rho} / g_{\rho}$, and that experimental data are described best with zero tensor coupling. However, a softer $\rho \pi \gamma$ form factor than that of Eq. (82)

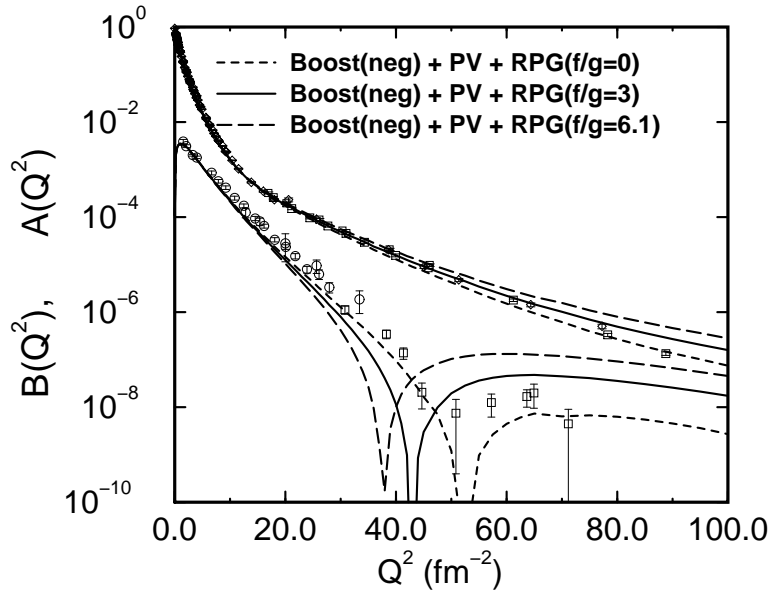

FIG. 7: The deuteron structure functions $A$ and $B$. Each line is based on including all positive and negative-energy states, the boost, the PV-coupling MEC and the $\rho \pi \gamma$ MEC. Lines differ only by the value of tensor $\rho N$ coupling $f_{\rho} / g_{\rho}$ used in the $\rho \pi \gamma$ graph.

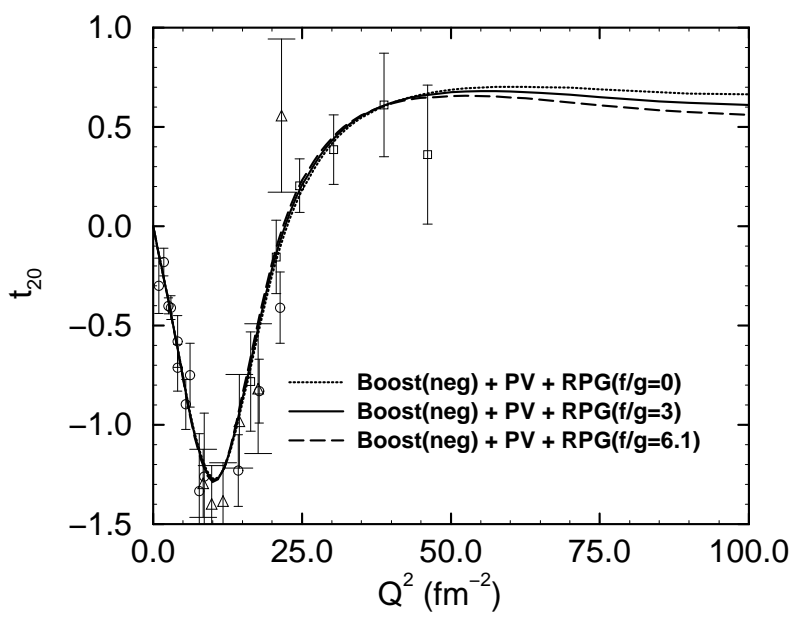

FIG. 8: The tensor-polarization observable $t_{20}$. Each line is based on including all positive and negative-energy states, the boost, the PV-coupling MEC and the $\rho \pi \gamma$ MEC. Lines differ only by the value of tensor $\rho N$ coupling $f / g$ used in the $\rho \pi \gamma$ graph.

would reduce the impact of the $\rho \pi \gamma$ MEC on all observables. Also, Hummel and Tjon 19] have estimated the effects of a possible $\omega \sigma \gamma$ meson-exchange current and found that it can have a significant effect on the minimum of the $B$ observable when a vector-meson-dominance $\omega \sigma \gamma$ form factor is used. Clearly, a better understanding of the effects of meson-exchange corrections on the electrondeuteron $B$ structure function is needed. Lattice QCD calculations along the lines of Ref. [46] are needed for the $\rho \pi \gamma$ form factor with $Q^{2}$ values up to $2 \mathrm{GeV}^{2}$. 
Negative-energy states have significant effects on $A$ and (particularly) B. See, e.g. the curves labeled Boost $(++)$ and Boost(neg) in Fig. 3. We do not, however, find as large an effect from negative-energy states as was obtained in the work of van Orden, Devine and Gross [20]. There the inclusion of such effects produced an acceptable description of the $B$ data. This is likely due in part to the fact that we use a pseudovector $\pi N$ coupling, whereas in Ref. 20] an admixture of about 25\% pseudoscalar $\pi N$ coupling was employed. Pseudoscalar $\pi N$ coupling is known to produce larger effects from negativeenergy states.

In order to clarify the significance of including all positive and negative-energy states in our analysis, we also calculate the leading-order Z-graphs. Figs. 9] and [10 compare results obtained using ++ states only, adding the leading-order Z-graphs as in Eq. (84), and including all positive and negative-energy states. Meson-exchange currents are omitted. In the case of observable $B$ the leading-order Z-graphs do not accurately reproduce the results of a calculation which includes all positive- and negative-energy states to all orders in perturbation theory. First-order perturbation theory for Z-graphs is adequate for observables $A$ and $t_{20}$, but not for $B$. Solving for the deuteron vertex functions non-perturbatively in all positive- and negative-energy sectors one finds smaller effects on the $B$ observable due to couplings to negativeenergy states than are predicted by leading-order Zgraphs.

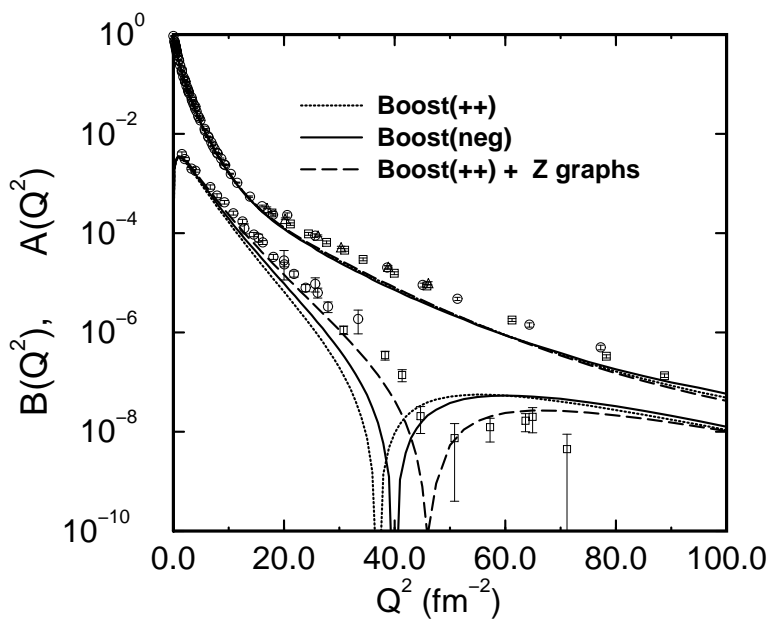

FIG. 9: The deuteron structure functions $A$ and $B$. In all cases the boost is included and the meson-exchange corrections are omitted. Dotted lines are based on including ++ states only, solid lines are based on including all positive and negative-energy states and dashed lines are based on including ++ states and adding the $\mathrm{Z}$ graphs in first-order perturbation theory as in Eq. 84).

Our results suggest that the existing data for the deuteron's $A$ and $t_{20}$ observables are described reasonably well when relativistic dynamics, boost effects and

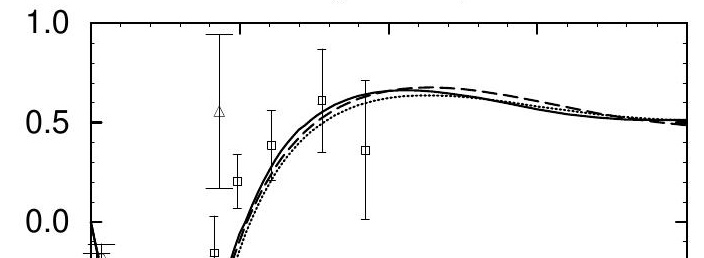

the PV-coupling current are implemented in a consistent formalism and calculation. However, the data for the $B$ observable are not explained unless there is some non-standard contribution, such as the $\rho \pi \gamma$ MEC with $f_{\rho}=0$. Other analyses have suggested that various relativistic effects, effects of negative-energy states or effects of the PV-coupling current might be larger than we find, but we believe our examination of each of these effects to be reliable within the ET formalism.

If a vector-meson-dominance $\rho \pi \gamma$ form factor is correct, then the $\rho \pi \gamma$ MEC can help to explain the $B$ data provided that a very small tensor $\rho N$ coupling is used. The ratio $f_{\rho} / g_{\rho}$ should be substantially less than the 6.1 used in one-boson-exchange models of the $N N$ interaction, or even a value which would explain the isovector magnetic moment of the nucleon. Fig. 8 shows that the $t_{20}$ data of Refs. 1, 58, 59, 60 are well-described by our approach out to $Q^{2} \approx 2 \mathrm{GeV}^{2}$. This observable is fairly insensitive to some of the dynamics that plays a role in $A$ and $B$ (single-nucleon form factors, the $\rho \pi \gamma$ MEC). However, it is quite sensitive to relativistic effects (see, for instance 8,23$]$ ), so it is gratifying that our approach reproduces the data, especially that of Ref. 1] at large $Q^{2}$, so well.

\section{Acknowledgments}

We thank J. Adam, E. J. Beise, R. Edwards, M. Petratos, and J. A. Tjon for useful conversations. D. R. P. thanks both the Theory Group at Jefferson Lab and the TQHN group at the University of Maryland for their hospitality during this work. We are also grateful to the U. S. Department of Energy, Nuclear Physics Division, for its support under grants DE-FG02-93ER-40756, DE-FG02-02ER41218, DE-FG02-93ER-40762, and DEFG03-97ER41014. 
[1] D. Abbott et al. [JLAB t(20) Collaboration], Phys. Rev. Lett. 84, 5053 (2000) arXiv:nucl-ex/0001006.

[2] D. Abbott et al. [JLAB t20 Collaboration], Eur. Phys. J. A 7, 421 (2000) arXiv:nucl-ex/0002003.

[3] L. C. Alexa et al., Phys. Rev. Lett. 82, 1374 (1999).

[4] D. Abbott et al., Phys. Rev. Lett. 82, 1379 (1999).

[5] M. Garcon and J. W. van Orden, Adv. Nucl. Phys. 26, 293 (2001).

[6] R. Gilman and F. Gross, J. Phys. G28, R37-R116 (2002).

[7] R. Schiavilla and D. O. Riska, Phys. Rev. C 43, 437 (1991).

[8] R. B. Wiringa, V. G. J. Stoks, and R. Schiavilla, Phys. Rev. C 51, 38 (1995).

[9] R. Schiavilla and V. R. Pandharipande, Phys. Rev. C65, 064009 (2002)

[10] H. Arenhovel, F. Ritz and T. Wilbois, Phys. Rev. C 61, 034002 (2000) arXiv:nucl-th/9910009.

[11] I. Sick, Prog. Part. Nucl. Phys. 47, 245-318 (2001).

[12] S. Weinberg, Phys. Lett. B. 251, 288 (1990).

[13] S. Weinberg, Nucl. Phys. B363, 3 (1991).

[14] S. Weinberg, Phys. Lett. B. 295, 114 (1992).

[15] D. R. Phillips, Phys. Lett. B 567, 12 (2003) arXiv:nucl-th/0304046.

[16] D. R. Phillips and S. J. Wallace, Phys. Rev. C 54, 507 (1996).

[17] D. R. Phillips and S. J. Wallace, Few-body Syst. 24, 175 (1997).

[18] D. R. Phillips, S. J. Wallace, and N. K. Devine, Phys. Rev. C 58, 2261 (1998).

[19] E. Hummel and J. A. Tjon, Phys. Rev. Lett. 63, 1788 (1989); Phys. Rev. C 42, 423 (1990); ibid. 49, 21 (1994).

[20] J. W. Van Orden, N. K. Devine, and F. Gross, Phys. Rev. Lett. 75, 4369 (1995).

[21] R. G. Arnold, C. E. Carlson, and F. Gross, Phys. Rev. C 21, 1426 (1980).

[22] P. L. Chung, F. Coester, and B. D. Keister, and W. N. Polyzou, Phys. Rev. C 37, 2000 (1988).

[23] J. Carbonell and V. A. Karmanov, Eur. Phys. J. A 6, 9 (1999).

[24] J. Carlson and R. Schiavilla, Rev. Mod. Phys. 70, 743 (1998).

[25] R. Machleidt, Adv. Nucl. Phys. 19, 189 (1989).

[26] S. J. Wallace, Phys. Rev. Lett. 87, 180401-1 (2001)

[27] F. Coester and W. Polyzou, Phys. Rev. D 1348 (1982).

[28] B. Bakamjian and L. H. Thomas, Phys. Rev. 92, 1300 (1953).

[29] D. O. Riska, Prog. Part. Nucl. Phys. 11, 199 (1984).

[30] J. L. Friar, Ann. Phys. 104, 380 (1977); Phys. Rev. C 22, 796 (1980).

[31] J. Adam, H. Goller, and H. Arenhövel, Phys. Rev. C 48, 370 (1993).

[32] J. Fleischer and J. A. Tjon, Nucl. Phys. B84, 375 (1975); Phys. Rev. D 15, 2537 (1977); ibid. 21, 87 (1980).

[33] F. Gross, Phys. Rev. C 26, 2203 (1982).

[34] E. E. Salpeter, Phys. Rev. 87, 328 (1952).

[35] A. A. Logunov and A. N. Tavkhelidze, Nuovo Cim. 29,
380 (1963).

[36] A. N. Kvinikhidze and B. Blankleider, Few-Body Syst. Suppl. 7, 294 (1994).

[37] A. Klein, Phys. Rev. 90, 1101 (1953); ibid. 94, 1052 (1954).

[38] V. B. Mandelzweig and S. J. Wallace, Phys. Lett. B. 197, 469 (1987); S. J. Wallace and V. B. Mandelzweig, Nucl. Phys. A503, 673 (1989).

[39] D. B. Kaplan, M. J. Savage and M. B. Wise, Nucl. Phys. B 534, 329 (1998).

[40] F. Gross and D. O. Riska, Phys. Rev. C 36, 1928 (1987).

[41] P. A. M. Dirac, Rev. Mod. Phys. 21, 392 (1949).

[42] Strict expansions in powers of $1 / m$ should be avoided because corrections like $p^{4} / m^{4}$ are not bounded as $p \rightarrow$ $\infty$. Our expansions generally involve bounded operators. We refer to typical bounded correction terms, such as $p^{4} / \epsilon^{4}$, as order $1 / m^{4}$ for simplicity.

[43] J. J. Adam and H. Arenhovel, Nucl. Phys. A 614, 289 (1997)

[44] T.-S. Park, D.-P. Min, and M. Rho, Nucl. Phys. A596, 515 (1996).

[45] E. Truhlik, J. Smejkal and F. C. Khanna, Nucl. Phys. A689, 741 (2001).

[46] R. G. Edwards, hep-lat/0409119

[47] H. Ito and F. Gross, Phys. Rev. Lett. 71, 2555 (1993).

[48] M. D. Cozma, "Proton-proton bremsstrahlung and elastic nucleon-nucleon scattering: relativistic formulations", Ph. D. thesis submitted to University of Groningen (2004).

[49] R. K. Bhaduri, "Models of the Nucleon" (AddisonWesley, Reading MA, 1998), p. 271.

[50] V. G. J. Stoks, R. A. M. Klomp, C. P. F. Terheggen, and J. J. de Swart, Phys. Rev. C 49, 2950 (1994).

[51] R. Machleidt, F. Sammarucca, and Y. Song, Phys. Rev. C 53, R1483 (1996).

[52] J. J. Kelly, Phys. Rev. C 66, 065203 (2002); and submitted to Phys. Rev. C, September 2004.

[53] P. Mergell, U.-G. Meissner, and D. Drechsel, Nucl. Phys. A596, 367 (1996).

[54] J. E. Elias et al., Phys. Rev. 177, 2075 (1969); R. G. Arnold et al., Phys. Rev. Lett. 35, 776 (1975); S. Platchkov et al., Nucl. Phys. A510, 740 (1990).

[55] G. G. Simon, C. Schmitt, and V. H. Walther, Nucl. Phys. A364, 285 (1981); R. Cramer et al., Z. Phys. C 29, 513 (1985).

[56] S. Auffret et al., Phys. Rev. Lett. 54, 649 (1985).

[57] P. Bosted et al., Phys. Rev. C 42, 38 (1990).

[58] M. E. Schulze et al., Phys. Rev. Lett. 52, 597 (1984); V. F. Dmitriev et al., Phys. Lett. B. 157, 143 (1985); B. B. Voitsekhovskii et al., JETP Lett. 43, 733 (1986); R. Gilman et al., Phys. Rev. Lett. 65, 1733 (1990); M. Ferro-Luzzi et al., Phys. Rev. Lett. 77, 2630 (1996); I. The et al., Phys. Rev. Lett. 67, 173 (1991).

[59] M. Bouwhuis et al. Phys. Rev. Lett. 82, 3755 (1999).

[60] D. M. Nikolenko et al. Phys. Rev. Lett. 90, 072501. 